\title{
Rotational properties of hypermassive neutron stars from binary mergers
}

\author{
Matthias Hanauske, ${ }^{1,2}$ Kentaro Takami, ${ }^{3,1}$ Luke Bovard, ${ }^{1}$ Luciano Rezzolla,,${ }^{1,2}$ \\ José A. Font, ${ }^{4,5}$ Filippo Galeazzi, ${ }^{1}$ and Horst Stöcker ${ }^{1,2,6}$ \\ ${ }^{1}$ Institut für Theoretische Physik, Max-von-Laue-Straße 1, 60438 Frankfurt, Germany \\ ${ }^{2}$ Frankfurt Institute for Advanced Studies, Ruth-Moufang-Straße 1, 60438 Frankfurt, Germany \\ ${ }^{3}$ Kobe City College of Technology, 651-2194 Kobe, Japan \\ ${ }^{4}$ Departamento de Astronomía y Astrofísica, Universitat de València, Dr. Moliner 50, 46100, Burjassot (València), Spain \\ ${ }^{5}$ Observatori Astronòmic, Universitat de València, C/ Catedrático José Beltrán 2, 46980, Paterna (València), Spain \\ ${ }^{6}$ GSI Helmholtzzentrum für Schwerionenforschung GmbH, 64291 Darmstadt, Germany
}

\begin{abstract}
Determining the differential-rotation law of compact stellar objects produced in binary neutron stars mergers or core-collapse supernovae is an old problem in relativistic astrophysics. Addressing this problem is important because it impacts directly on the maximum mass these objects can attain and hence on the threshold to blackhole formation under realistic conditions. Using the results from a large number of numerical simulations in full general relativity of binary neutron star mergers described with various equations of state and masses, we study the rotational properties of the resulting hypermassive neutron stars. We find that the angular-velocity distribution shows only a modest dependence on the equation of state, thus exhibiting the traits of "quasiuniversality" found in other aspects of compact stars, both isolated and in binary systems. The distributions are characterized by an almost uniformly rotating core and a "disk". Such a configuration is significantly different from the $j$-constant differential-rotation law that is commonly adopted in equilibrium models of differentially rotating stars. Furthermore, the rest-mass contained in such a disk can be quite large, ranging from $\simeq 0.03 M_{\odot}$ in the case of high-mass binaries with stiff equations of state, up to $\simeq 0.2 M_{\odot}$ for low-mass binaries with soft equations of state. We comment on the astrophysical implications of our findings and on the long-term evolutionary scenarios that can be conjectured on the basis of our simulations.
\end{abstract}

PACS numbers: $04.25 . \mathrm{Dm}$, 04.25.dk, 04.30.Db, 04.40.Dg, 95.30.Lz, 95.30.Sf, 97.60.Jd

\section{INTRODUCTION}

A number of catastrophic astrophysical events, such as the collapse of the iron core in Type-II supernovae or the merger of a binary system of neutron stars, lead to the formation of a compact object with a large amount of angular momentum. Clearly, uniform rotation is not an efficient way of sustaining such large rotation rates and it is far easier to obtain an equilibrium by distributing the angular momentum differentially in radius. At the same time, for a given amount of mass involved in the event, the knowledge of the law of differential rotation is important in establishing how close or how far the equilibrium configuration attained is from the instability threshold of the gravitational collapse to a black hole. On the basis of these considerations, it is clear that the problem of determining the law of differential rotation produced in these events is an important one.

Over the last 15 years, a large number of works have explored this problem in full general relativity, either through the construction of equilibrium configurations [1-8] or through their dynamical production in core-collapse supernovae (see [9] for an overview) or in binary neutron star mergers (see [10] for a recent review). Lacking a physically motivated law of differential rotation, essentially all of these works have assumed that the rotation law is particularly simple and such that the specific angular momentum $j:=h u_{\phi}$ is constant, with $h$ being the specific enthalpy and $u_{\phi}$ the covariant azimuthal component of the four-velocity. This law obviously satisfies the Rayleigh criterion for local dynamical stability against axisymmetric perturbations, $d j / d \Omega<0$, where $\Omega$ is the angular velocity. More importantly, however, it has the ad- vantage of being analytically simple, with the angular velocity decreasing monotonically from the center of the star and with the degree of differential rotation being regulated by a single dimensionless parameter (normally referred to as $\tilde{A}$ ). Only recently, Ref. [6] has made the first attempts to generalize the space of equilibria by considering more general prescriptions for the law of differential rotation, using however analytical simplicity as the guideline.

Overall, all the studies carried out so far on equilibrium sequences of stationary models agree that when using a $j-$ constant law of differential rotation, several different solutions are possible for a given degree of differential rotation $[5,8]$ and that the maximum mass depends on both the degree of differential rotation and on the type of solution, reaching values as large as four times that of the maximum mass of nonrotating configurations $M_{\text {тог }}[5,8]$. This is to be contrasted with what happens in the case of uniformly-rotating models, where the maximum mass has recently been found to be only $20 \%$ larger than $M_{\text {то }}$, quite independently of the equation of state (EOS) [11].

While the $j$-constant law of differential rotation has been useful so far to explore the equilibria of differentially rotating compact stars, it is also hard to justify on physical grounds, in particular when considering the results of numerical simulations of merging binary neutron stars. A number of studies in this direction, in fact, have shown that although the merger normally leads to the formation of a hypermassive neutron star (HMNS), that is, a neutron star whose mass exceeds the maximum mass of a uniformly rotating star, the law of differential rotation is rather different [12-18]. Hence, the need to determine a law of differential rotation that, albeit not simpler, 
does reflect the hydrodynamical equilibrium that is attained in HMNSs produced in binary neutron-star mergers. Clearly, determining such a law of differential rotation is important since it has direct impacts on the maximum mass these objects can attain and hence on the threshold to black-hole formation under astrophysically realistic conditions.

A first step in this direction has been taken in Refs. [19, 20], where the properties of the angular-velocity distribution in the merged object have been analyzed in detail, although only for a very limited number of binaries. In agreement with the previous simulations of Ref. [4], these more recent works have found that the angular-velocity profile of the HMNS shows a slowly rotating core and an envelope that rotates at angular frequencies that scale as $r^{-3 / 2}$, where $r$ is the radial coordinate in our coordinate system. This holds true both when the initial data is that of irrotational binaries and when the binaries are artificially spun-up as done in Ref. [21].

Here, we adopt a more systematic approach and study the rotational properties of the HMNSs produced by the merger of binary neutron stars described with various EOSs and masses, and as computed via a large number of numerical simulations in full general relativity. Besides confirming the more specific results of Refs. [4, 19, 20], our most interesting finding is arguably that the angular-velocity distribution shows only a modest dependence on the EOS, thus exhibiting the traits of "quasi-universality" that have been found in other aspects of compact stars, both when isolated [11, 22-27] and in binary systems [22, 28-30]. More specifically, the EOS-independent angular-velocity distributions we find are characterized by an almost uniformly rotating core and a "disk" with angular frequencies $\Omega(r) \propto r^{-3 / 2}$.

Having a "disk" in the outer regions of the HMNS is important for two reasons, at least. Firstly, the disk surrounding the HMNS will accrete onto the uniformly rotating core of the star only on a dissipative timescale, thus not affecting its long-term stability. Secondly, once the core of the HMNS eventually collapses to a rotating black hole, the presence of a certain amount of mass on stable orbits will guarantee that the black hole will not be "naked", but surrounded by a torus, as expected when the collapse to a black hole is prompt [13]. Both of these considerations are important within the protomagnetar model for short gamma-ray bursts [31-33] and the subsequent extended X-ray emission [34] (see also [35] for a similar model).

The paper is organized as follows: Section II is dedicated to a brief overview of the mathematical and numerical setup employed in our simulations, while in Secs. III and IV we illustrate the results obtained when modelling high- and lowmass binaries, respectively. We focus, in particular, on the distributions of rest-mass density and angular velocity, and illustrate our approach to obtain time and azimuthally averaged profiles. Section $\mathrm{V}$ focuses on the use of tracer particles to disentangle the physically meaningful results from the possible contamination of gauge effects, while Sec. VI discusses the "quasi-universal" features of the angular-velocity profiles and how to correlate them with the properties of the progenitor stars in the binary. Also discussed in Sec. VI are the amount of mass in the disk and the influence of the thermal component of the EOS on the results presented. Finally, in Sec. VII we present a summary of our findings and the impact they have on the stability of differentially rotating compact stars. Appendix A provides a discussion in terms of the dynamics of tracers on the conservation of the Bernoulli constant in the quasi equilibrium of the HMNS. Appendix B provides a discussion of the effects of resolution and symmetries on the lifetime and evolution of the HMNS.

Hereafter, we will use a spacelike signature $(-,+,+,+)$ and a system of units in which $c=G=M_{\odot}=1$ unless stated differently.

\section{GENERAL FRAMEWORK}

\section{A. Mathematical and numerical setup}

The mathematical and numerical setup used for the simulations reported here is the same discussed in Refs. [29, 36] and presented in greater detail in other papers [13, 37, 38]. For completeness we review here only the basic aspects, referring the interested reader to the papers above for additional information.

Our simulations are performed in general relativity using the fourth-order finite-differencing code McLachlan [39, 40], which is part of the publicly available Einstein Toolkit [41]. This code solves a conformal traceless formulation of the Einstein equations [42-44], with a " $1+\log$ " slicing condition and a "Gamma-driver" shift condition [45, 46]. Correspondingly, the evolution of the general-relativistic hydrodynamics equations is done using the finite-volume code Whisky [47], which has been extensively tested in simulations involving the inspiral and merger of binary neutron stars [13, 37, 48, 49]. The hydrodynamics equations, expressing the conservation of energy, momentum and rest mass [50], are cast in the conservative Valencia formulation [51]. Their numerical solution is obtained employing the Harten-Lax-van Leer-Einfeldt [52] approximate Riemann solver [52] in conjunction with the Piecewise Parabolic Method [53] for the reconstruction of the evolved variables. For the time integration of the coupled set of hydrodynamic and Einstein equations we use the Method of Lines with an explicit fourth-order Runge-Kutta method. Our simulations use a CFL number of 0.35 to compute the timestep.

An adaptive mesh refinement (AMR) approach based on the Carpet mesh-refinement driver [54] is used to both increase resolution and extend the spatial domain, placing the outer boundary as close as possible to the wave zone. The grid hierarchy consists of six refinement levels with a grid resolution varying from $\Delta h_{5}=0.15 M_{\odot}$ (i.e., $\simeq 221 \mathrm{~m}$ ) for the finest level to $\Delta h_{0}=4.8 M_{\odot}$ (i.e., $\simeq 7.1 \mathrm{~km}$ ) for the coarsest level, whose outer boundary is at $514 M_{\odot}$ (i.e., $\simeq 759 \mathrm{~km}$ ). To reduce computational costs, a reflection symmetry across the $z=0$ plane and a $\pi$-symmetry condition across the $x=0$ plane has been adopted (see Appendix B for a discussion of the influence on the results of this resolution and of the symmetry assumed). The initial configuration for the quasi- 
equilibrium irrotational binary neutron stars has been generated with the use of the LORENE-code [55] and an initial coordinate separation of the stellar centers of $45 \mathrm{~km}$ has been used for all binaries. For each EOS, we have considered equalmass binaries where each star has initial gravitational masses that are either $M=1.25 M_{\odot}$ (low-mass binaries; cf. Sec. IV) or $1.35 M_{\odot}$ (high-mass binaries; cf. Sec. III) at infinite separation.

A quantity that is particularly important in our analysis is the angular velocity, $\Omega$, which is defined as the amount of coordinate rotation

$$
\Omega:=\frac{d \phi}{d t}=\frac{d x^{\phi}}{d t}=\frac{u^{\phi}}{u^{t}},
$$

where $u^{\phi}$ and $u^{t}$ are components of the four-velocity vector $u^{\mu}$. The corresponding three-velocity as measured by the same Eulerian observer is then defined as

$$
v^{i}:=\frac{\gamma_{\mu}^{i} u^{\mu}}{-n_{\mu} u^{\mu}}=\frac{1}{\alpha}\left(\frac{u^{i}}{u^{t}}+\beta^{i}\right),
$$

where $\alpha$ is the lapse function, $\beta^{i}$ is the shift vector, $n^{\mu}$ is the unit timelike vector normal to a constant $t$ hypersurface, and $\gamma_{i j}$ is the three-dimensional metric. With the above definition the angular velocity within the $3+1$ split can be expressed as

$$
\Omega=\alpha v^{\phi}-\beta^{\phi},
$$

where

$$
\begin{aligned}
v^{\phi} & =\frac{x v^{y}-y v^{x}}{x^{2}+y^{2}+z^{2}}, \\
\beta^{\phi} & =\frac{x \beta^{y}-y \beta^{x}}{x^{2}+y^{2}+z^{2}},
\end{aligned}
$$

are the three-velocity and shift vector components as computed from the Cartesian grid with coordinates $(x, y, z)$. Written in this form, we can interpret $\Omega$ as consisting of a lapsecorrected part of the $\phi$-component of the three-velocity, minus a frame-dragging term provided by the $\phi$-component of the shift vector.

\section{B. Microphysical matter treatment}

To close the set of evolution equations an EOS is needed and which provides a relation among the thermodynamical properties of the neutron-star matter. A general EOS describes the pressure as a function of the rest-mass density, particle composition and temperature. However, for certain parts of the merger, simplified versions can be used. In particular, during the inspiral, a "cold", i.e., temperature independent, EOS is sufficient to represent the state of the neutron-star matter prior to merger. After contact, when the HMNS is formed, large shocks will increase the temperature and to account for this additional heating a full temperature dependent nuclearphysics EOS is required. As the number of such EOSs is unfortunately still limited, we include thermal effects by adding an ideal-fluid component that accounts for the shock heating.
The pressure $p$ and the specific internal energy $\epsilon$ are therefore composed of a cold nuclear-physics part and of a "thermal" ideal-fluid component ${ }^{1}$ [56]

$$
p=p_{\mathrm{c}}+p_{\mathrm{th}}, \quad \epsilon=\epsilon_{\mathrm{c}}+\epsilon_{\mathrm{th}},
$$

where $p$ and $\epsilon$ are the pressure and specific internal energy, respectively. We model the cold part $p_{\mathrm{c}}, \epsilon_{\mathrm{c}}$ with five different nuclear-physics EOSs. Two of such EOSs, namely APR4 [57] and SLy [58], belong to the class of variational-method EOSs and the underlying particle composition within these models consists mainly of neutrons with little admixtures of protons, electrons and muons. Additionally, two more EOSs, i.e., GNH3 [59] and H4 [60], are built using relativistic meanfield models which include, above a certain rest-mass density, hyperonic particles.

In contrast, the fifth EOS, namely ALF2 [61], is more a model for hybrid stars than for neutron stars because it implements a phase transition to color-flavor-locked quark matter. Within this model, the hadronic particles begin to deconfine to quark matter above a certain transition rest-mass density $\rho_{\text {trans }}=3 \rho_{\text {nuc }}$, where $\rho_{\text {nuc }}:=2.705 \times 10^{14} \mathrm{~g} / \mathrm{cm}^{3}$ is the nuclear-matter rest-mass density. Assuming a moderate surface tension of the quark matter droplets, a phase transition is implemented by using a Gibbs-construction. As charge neutrality is only globally conserved within this construction, a mixed-matter phase exists in the rest-mass density range $3 \rho_{\text {nuc }} \leq \rho \leq 7.8 \rho_{\text {nuc }}$.

We note that all of the EOSs used in our calculations satisfy the current observational constraint on the observed maximum mass in neutron stars, i.e., $2.01 \pm 0.04 M_{\odot}$ [62]. Instead of using the data tables of the various EOSs, we have found it more suitable to convert them to piecewise polytropes [63]. Each EOS has been parametrized, based on specifying its stiffness in three rest-mass density intervals $i=2,3,4$, measured by the adiabatic index $\Gamma_{i}=d \log \bar{p}_{i} / d \log \bar{\rho}_{i}$. Additionally, a unique polytrope with $\Gamma_{1}=1.357$ has been added for all of the used EOS to account for the star's low rest-mass density region $\rho \leq \rho_{\text {nuc }}$.

The "cold" nuclear-physics contribution to each EOS is obtained after expressing the pressure and specific internal energy $\epsilon_{\mathrm{c}}$ in the rest-mass density range $\rho_{i-1} \leq \rho<\rho_{i}$ as (for details see [36, 64-66])

$$
p_{\mathrm{c}}=K_{i} \rho^{\Gamma_{i}}, \quad \epsilon_{\mathrm{c}}=\epsilon_{i}+K_{i} \frac{\rho^{\Gamma_{i}-1}}{\Gamma_{i}-1} .
$$

For an overall consistency, the rest-mass density ranges used for the piecewise polytropes have been chosen to be the same for the different EOSs $\left(\rho_{2}=5.012 \times 10^{14} \mathrm{~g} / \mathrm{cm}^{3}\right.$ and $\rho_{3}=$ $10^{15} \mathrm{~g} / \mathrm{cm}^{3}$ ). The transition densities $\rho_{1}$ to the low rest-mass density polytrope and the EOS-dependent adiabatic indexes $\Gamma_{i}$ are summarized in Table 2 of [66]. Due to the implementation of the hadron-quark phase transition, the ALF2 EOS

\footnotetext{
1 This class of EOSs is referred in the literature as the so-called "hybrid EOS" [50] and should not be meant to indicate that the star is composed of a hybrid hadron-quark matter present in hybrid stars.
} 


\begin{tabular}{l|c|c|c|c|c|c|c|c|c|c|c}
\hline \hline model & EOS & $\begin{array}{c}M \\
{\left[M_{\odot}\right]}\end{array}$ & $\begin{array}{c}R \\
{[\mathrm{~km}]}\end{array}$ & $\begin{array}{c}M_{\mathrm{ADM}} \\
{\left[M_{\odot}\right]}\end{array}$ & $\begin{array}{c}M_{\mathrm{b}} \\
{\left[M_{\odot}\right]}\end{array}$ & $M / R$ & $\begin{array}{c}f_{\text {orb }} \\
{[\mathrm{Hz}]}\end{array}$ & $\begin{array}{c}J \\
{\left[M_{\odot}^{2}\right]}\end{array}$ & $I / M^{3}$ & $k_{2}$ & $\lambda / M^{5}$ \\
\hline GNH3-M125 & GNH3 & 1.250 & 13.817 & 2.4780 & 1.3464 & 0.13358 & 273.29 & 6.4067 & 18.890 & 0.11753 & 1842.4 \\
GNH3-M135 & GNH3 & 1.350 & 13.777 & 2.6746 & 1.4641 & 0.14468 & 281.58 & 7.2766 & 16.450 & 0.10841 & 1139.9 \\
\hline H4-M125 & H4 & 1.250 & 13.533 & 2.4780 & 1.3506 & 0.13638 & 273.25 & 6.4058 & 18.610 & 0.12361 & 1746.5 \\
H4-M135 & H4 & 1.350 & 13.550 & 2.6746 & 1.4687 & 0.14711 & 281.61 & 7.2770 & 16.344 & 0.11483 & 1111.1 \\
\hline ALF2-M125 & ALF2 & 1.250 & 12.276 & 2.4779 & 1.3672 & 0.15034 & 273.16 & 6.4014 & 16.455 & 0.13049 & 1132.6 \\
ALF2-M135 & ALF2 & 1.350 & 12.353 & 2.6746 & 1.4877 & 0.16136 & 281.42 & 7.2708 & 14.581 & 0.12037 & 733.63 \\
\hline SLY-M125 & SLy & 1.250 & 11.469 & 2.4779 & 1.3720 & 0.16092 & 273.04 & 6.3977 & 14.000 & 0.10266 & 634.27 \\
SLY-M135 & SLy & 1.350 & 11.465 & 2.6745 & 1.4946 & 0.17386 & 281.34 & 7.2663 & 12.309 & 0.092993 & 390.29 \\
\hline APR4-M125 & APR4 & 1.250 & 11.052 & 2.4779 & 1.3783 & 0.16700 & 273.05 & 6.3973 & 13.226 & 0.099787 & 512.14 \\
APR4-M135 & APR4 & 1.350 & 11.079 & 2.6746 & 1.5020 & 0.17992 & 281.37 & 7.2665 & 11.720 & 0.090990 & 321.78 \\
\hline LS220-M132 & LS220 & 1.319 & 12.775 & 2.6127 & 1.4360 & 0.15108 & 278.68 & 6.9891 & 15.113 & 0.099289 & 840.93 \\
LS220-M135 & LS220 & 1.350 & 12.750 & 2.6740 & 1.4733 & 0.15638 & 281.29 & 7.2656 & 14.112 & 0.096575 & 688.26 \\
\hline \hline
\end{tabular}

TABLE I. All binaries evolved and their properties. The various columns denote the gravitational mass $M$ of the binary components at infinite separation, the corresponding radius $R$, the ADM mass $M_{\mathrm{ADM}}$ of the binary system at the initial separation, the baryon mass $M_{\mathrm{b}}$, the compactness $\mathcal{C}:=M / R$, the orbital frequency $f_{\text {orb }}$ at the initial separation, the total angular momentum $J$ at the initial separation, the dimensionless moment of inertia $I / M^{3}$ at infinite separation, the $\ell=2$ dimensionless tidal Love number $k_{2}$ at infinite separation, and the dimensionless tidal deformability $\lambda / M^{5}$ defined by $\lambda:=2 k_{2} R^{5} / 3$.

has the largest softening at the rest-mass density boundary $\rho_{2}$ $\left(\Gamma_{1}=4.070\right.$ and $\left.\Gamma_{2}=2.411\right)$. Finally, the "thermal" part of the EOS is given by

$$
p_{\mathrm{th}}=\rho \epsilon_{\mathrm{th}}\left(\Gamma_{\mathrm{th}}-1\right), \quad \epsilon_{\mathrm{th}}=\epsilon-\epsilon_{\mathrm{c}} .
$$

where the last equality in (8) is really a definition, since $\epsilon$ refers to the computed value of the specific internal energy. In all of the simulations reported hereafter we use $\Gamma_{\text {th }}=2.0$ (see $[36,66]$ for an analysis of the effect of different $\Gamma_{\text {th }}$ and Sec. VIC for a discussion of the impact of $\Gamma_{\text {th }}$ on the results presented here). Detailed information on all the binaries and their properties is collected in Table I.

Finally, we note that in order to verify our hybrid-EOS approach and numerical setup and contrast the results with an alternative one, an additional simulation has been performed using the "hot", i.e., temperature dependent, LattimerSwesty (LS220) EOS [67]. For this simulation the hydrodynamic equations are solved employing the WhiskyTHC code $[68,69]$ and the BSSNOK formulation of the Einstein equations [70].

\section{HIGH-MASS BINARIES}

The main results concerning the evolution of the characteristic properties of the HMNSs are presented in this and the following section. To explain the procedure of how the HMNS properties and rotational profiles are calculated in our approach, we start by describing in this section the dynamics of a representative "high-mass binary" focusing on the ALF 2-M1 35 run, that is, a binary described by the ALF2 EOS and with total gravitational mass of $2 \times 1.35 M_{\odot}$; in the following section, we describe instead the ALF2-M125 run as an illustrative case of "low-mass simulations".

We recall that with the exception of the APR4 and LS220 EOSs, four of the six binaries with high masses $(M=$

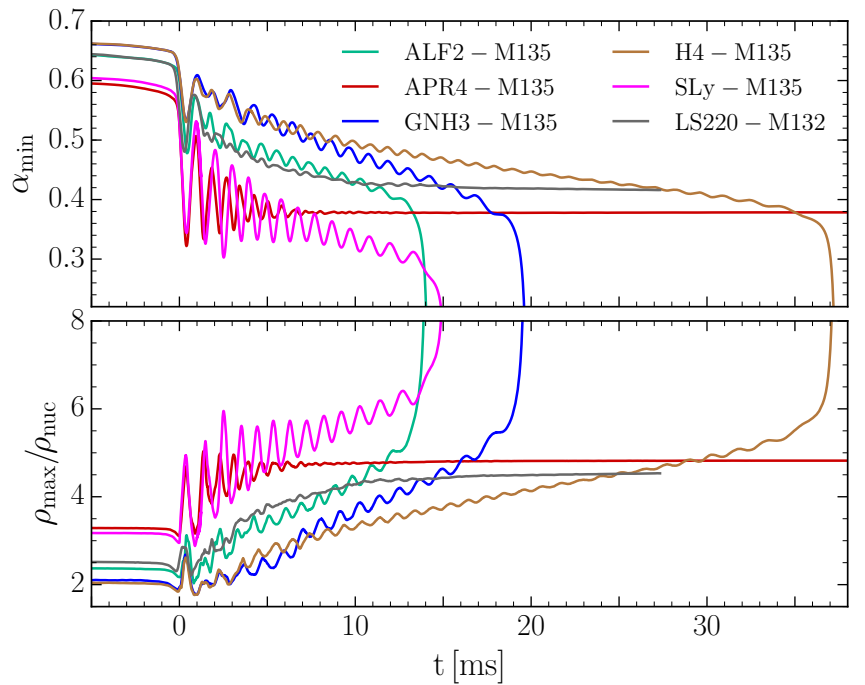

FIG. 1. Minimum value of the lapse function $\alpha_{\min }$ (upper panel) and maximum of the rest-mass density $\rho_{\max }$ in units of the nuclearmatter rest-mass density $\rho_{\text {nuc }}$ (lower panel) versus time in milliseconds after the merger for the high-mass simulations. All models collapse to a black hole except for the APR4 and LS220 EOSs.

$\left.1.35 M_{\odot}\right)$ collapse to a black hole within the simulated timescale, while for the low-mass cases $\left(M=1.25 M_{\odot}\right)$ none of the simulations show a gravitational collapse within our simulation time domain (see Figs. 1 and 7).

Before the merger, the maximum (central) value of the restmass density $\rho_{\max }$ is essentially constant in time and the minimum (central) value of the lapse function $\alpha$ decreases only slightly (see Fig. 1 for $t<0$, where we define as $t=0:=t_{\mathrm{M}}$ the time of merger or, equivalently, the time of the first maximum in the gravitational-wave amplitude). The differences 


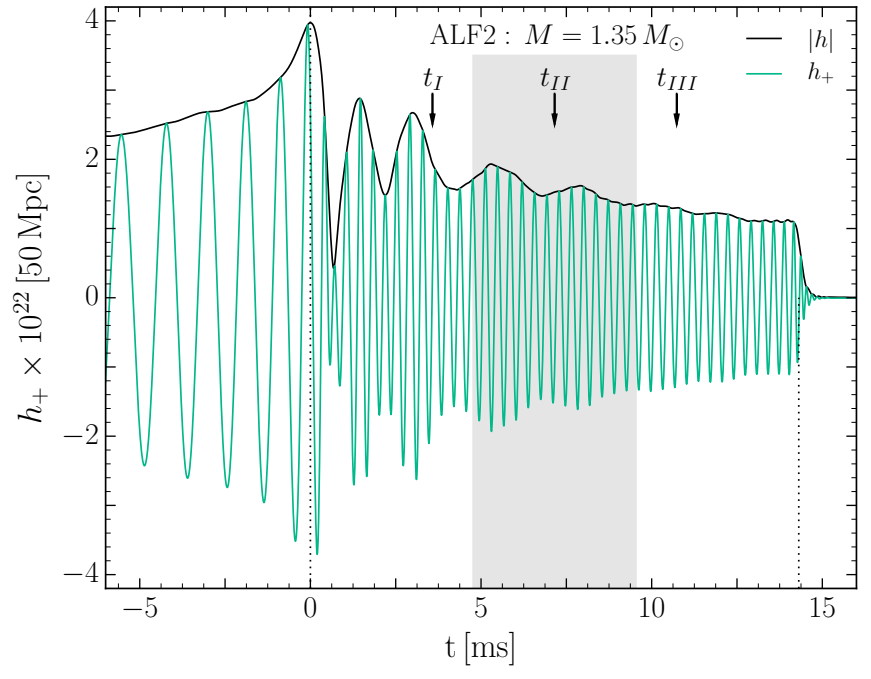

FIG. 2. Gravitational-wave amplitude $|h|$ (black line) and strain amplitude in the + polarisation $h_{+}$(green line) for the ALF2-M135 binary at a distance of $50 \mathrm{Mpc}$. Shaded in gray is the portion where a time average is performed, while the arrows indicate the times when representative distributions of the rest-mass density and angular velocity are shown in Fig. 3. Finally, the dotted vertical lines mark the time of merger and the first detection of an apparent horizon.

of the values of $\rho_{\max }$ and $\alpha_{\min }$ before merger are due to the softness/stiffness properties of the underlying EOSs, which determine the HMNS individual compactness. Although the HMNS is formed at the merger time, the rest-mass density profile at $t_{\mathrm{M}}=0$ still has two distinct maxima that correspond to the tidally deformed individual stars of the late inspiral phase. In the transient post-merger phase (i.e., $t \in[0,4] \mathrm{ms}$ ) irregular and strong fluctuations of $\rho_{\max }$ and $\alpha_{\min }$ occur, which are due to the violent and shock-dominated dynamics right after the merger. Within this early post-merger stage, the rest-mass density profiles of the HMNSs have two distinct maxima (the so-called "double-core" structure), which indicates that $\rho_{\max }$ and $\alpha_{\min }$ no longer correspond to the central HMNS values, respectively (see Ref. [66] for a simple mechanical toy model that describe this phase of the postmerger).

At later times (i.e., $t \gtrsim 4 \mathrm{~ms}$ ) the two maxima merge to one single maximum at the HMNS's center. This feature holds for all performed simulations with different EOSs and masses, and is in accordance with many other works (e.g., [13, 71]). Within this "post-transient" phase, $\rho_{\max }\left(\alpha_{\min }\right)$ show a quite regular oscillating behaviour with an average increasing (decreasing) value. Additionally, it is possible to note a continuous decrease in the oscillation frequency, which signals the approaching of the "zero-frequency" limit and hence the quasi-radial stability limit to gravitational collapse [72]. The lifetime of the HMNSs is different for the various EOSs and the collapse to the final black hole can be easily seen in Fig. 1 as a sudden singular increase in $\rho_{\max }$ (sudden decrease in $\left.\alpha_{\min }\right)$.

\section{A. Density evolution and gravitational-wave emission}

Before merger, the two individual stars of the ALF2-M135 binary have a central rest-mass density $\rho_{\max } \simeq 2.17 \rho_{\text {nuc }}$ that is below the onset of a hadron-quark phase transition, $\rho<3 \rho_{\text {nuc }}$. Hence, all of the matter inside the HMNS at merger time is mainly composed of neutrons with little admixtures of protons and electrons [61]. Within the early transient post-merger phase, $\rho_{\max }$ reaches values above $3 \rho_{\text {nuc }}$, but due to the strongly oscillating nature of the double core structure, the deconfined mixed phase does not remain in the HMNS and confines again to hadronic matter. Nevertheless, a considerable amount of hadronic matter deconfines in this transient post-merger phase to quark matter and in less than $0.5 \mathrm{~ms}$ it confines again to hadronic matter. We recall that under the "strange matter hypothesis", the strange-quark phase is the true ground state of matter and the whole neutron star would then transform into a pure-quark star after exceeding a certain deconfinement barrier [73-76]. During such a process a significant amount of energy would be released in the form of neutrinos and gamma-rays [77]. The quark-matter nucleation process, which takes place in the highly dynamical interior region of the HMNS, depends on various poorly known factors (e.g., the surface tension). In the ALF2 EOS, the strange-matter hypothesis has not been adopted and as a consequence, stable hybrid stars can be formed, having a inner core of deconfined strange matter.

In some other EOS-models of this kind, e.g., [78-82], there exist however parameter ranges where "twin stars" solutions are present [73] and transitions between these twins could likewise produce neutrino and even short gamma-ray bursts, which we do not account for in these simulations. Approximately $5 \mathrm{~ms}$ after merger, $\rho_{\max }$ is permanently above the phase-transition threshold and the HMNS contains an inner region of deconfined mixed-phase matter. A pure-quark phase appears only during the short collapse phase to black hole and finally this free quark matter will be macroscopically deconfined by the growth of the event horizon inside the collapsing HMNS.

For the gravitational-wave amplitudes $h_{+}$and $h_{\times}$we consider only the $(\ell, m)=(2,2)$ mode, which has been found to be the most dominant (for details see $[66,83]$ ). Figure 2 shows the gravitational-wave amplitude $|h|:=\left(h_{+}^{2}+h_{\times}^{2}\right)^{1 / 2}$ and $h_{+}$at a distance of $50 \mathrm{Mpc}$ as a function of time. The absolute maximum of $|h|$ corresponds to the time of merger for all of the different simulation runs. The last peak of $h_{+}$ corresponds approximately to the time when the black hole is formed (i.e., $t_{\mathrm{BH}}=14.16 \mathrm{~ms}$ ), which we have defined as the time when the apparent horizon is first detected. In order to compare the structural properties of the different HMNSs in the post-merger phase, we will later define a time-averaging procedure of the rotation profiles. This averaging time interval is already shown in Fig. 2 as a gray region.

The upper row of panels in Fig. 3 shows the evolution of the HMNS rest-mass density during the post-merger phase. The three different snapshots have been taken at $t=1 / 4,1 / 2$ and $3 / 4 t_{\mathrm{BH}}$. The left panel, in particular, which visualizes the rest-mass density distribution at $t_{\mathrm{I}}=t_{\mathrm{BH}} / 4 \approx 3.6 \mathrm{~ms}$, 

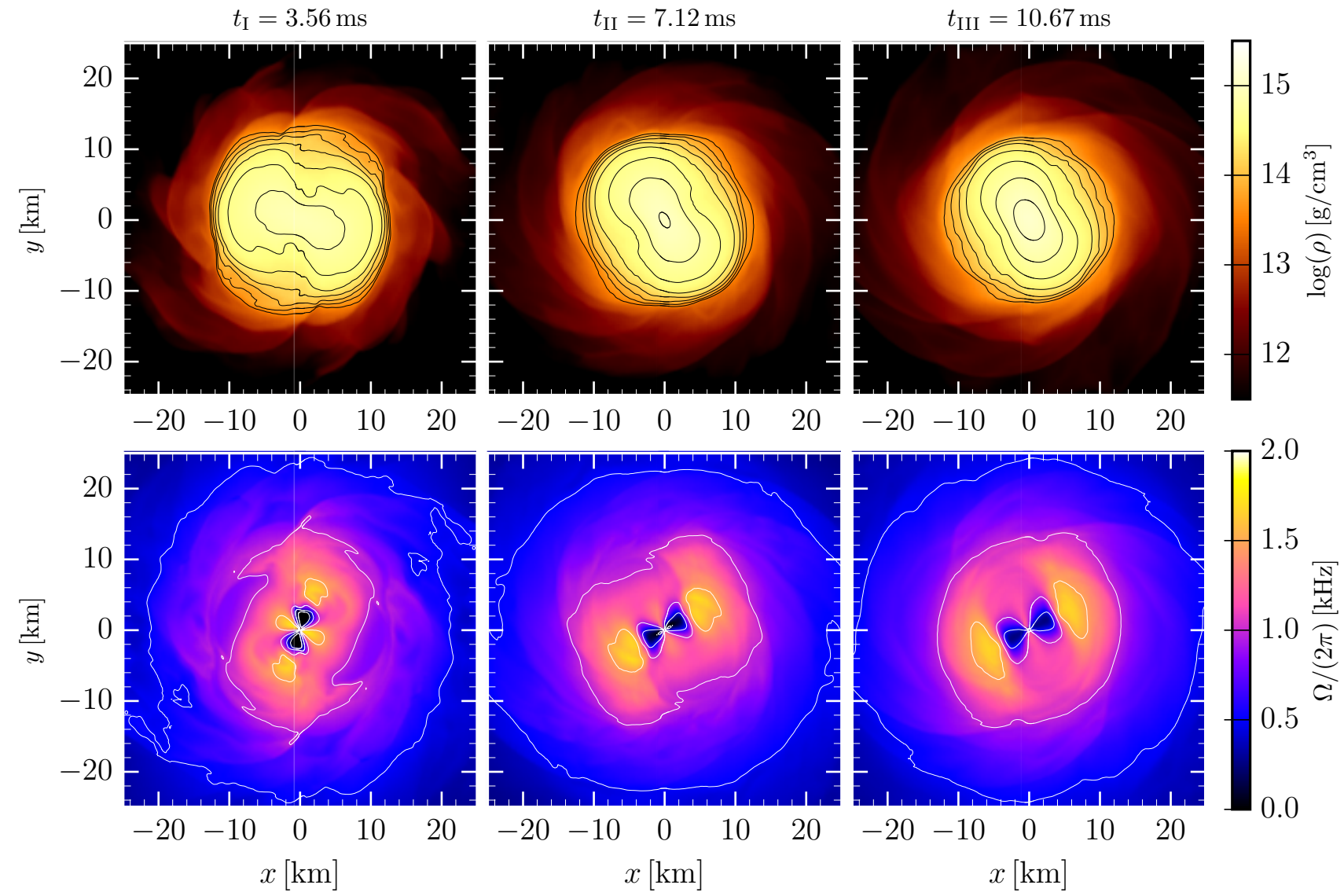

FIG. 3. Distributions of the rest-mass density (upper row, log scale) and of the fluid angular velocity (lower row) in the $(x, y)$ plane for the ALF 2-M135 binary at three different post-merger times as indicated in Fig. 2. The isocontours have been $\operatorname{drawn}$ at $\log (\rho)=13.6+0.2 n$ (upper row) and $\Omega=\{0,0.5,1.0,1.5,2.0\} \mathrm{kHz}$ (lower row), $n \in \mathbb{N}$.

shows that the overall rest-mass density of the HMNS is much higher than the rest-mass density at merger time. The doublecore structure in the inner area of the HMNS is right on the verge of merging to a single core and the maximum rest-mass density reached, $\rho_{\max } \simeq 3.1 \rho_{\text {nuc }}$, is slightly above the onset of the underlying hadron-quark phase transition. In the central and right upper panels of Fig. $3\left(t_{\mathrm{II}}=t_{\mathrm{BH}} / 2 \approx 7.1 \mathrm{~ms}\right.$ and $t_{\mathrm{III}}=3 t_{\mathrm{BH}} / 4 \approx 10.7 \mathrm{~ms}$ ) the double-core structure is no longer present. The value of the central rest-mass density maximum $\rho_{c} \simeq 3.8 \rho_{\text {nuc }}$ at $t_{\mathrm{II}}$ is clearly above the onset of the underlying hadron-quark phase transition. Since the ALF2 EOS uses a Gibbs construction for the modelling of the phase transition (see Section II), the inner core $(r \lesssim 3 \mathrm{~km})$ of the HMNS contains a crystalline structure of mixed phase matter, which might have additional effects on the evolution of the HMNS.

\section{B. Angular-velocity evolution: 2D slices}

In the following, and to contrast the description made above of the rest-mass density, we will concentrate on the evolution in the post-merger phase of the angular velocity $\Omega$ [see
Eq. (3)], which plays a particularly important role in our analysis. The lower panels of Fig. 3 display the distribution of $\Omega$ in the $(x, y)$ plane at the same times shown for the rest-mass density in the upper panels. In the first two milliseconds after the merger the time variation of $\Omega$ is very rapid, with two inner maxima placed between the double-core rest-mass density maxima (left lower panel). In the intermediate part of the post-merger (i.e., for $t \in\left[3 \mathrm{~ms}, t_{\mathrm{BH}}\right]$ ), $\Omega$ has a roughly time independent global structure in a frame corotating at half the frequency of the gravitational-wave emission, $\Omega_{\mathrm{GW}}$ (see Sec. V); this structure remains stationary for several milliseconds (right lower panel). Approximately two milliseconds before black hole formation, $\Omega$ largely increases in the center of the HMNS (not shown in Fig. 3.)

Note that also the angular-velocity distribution exhibits a clear $m=2$ distribution, where the two maxima rotate with the same frequency $\simeq 1.4 \mathrm{kHz}$ around the center of the HMNS as the non-axisymmetric $m=2$ perturbations of the rest-mass density (see upper middle panel of Fig. 3). Both maxima appear at a radial distance of $\simeq 6 \mathrm{~km}$, which is still clearly within the star's high rest-mass density range, but are outside the region where the hadron-quark phase transition appears. The maxima in $\Omega$ are also accompanied by two minima in the in- 

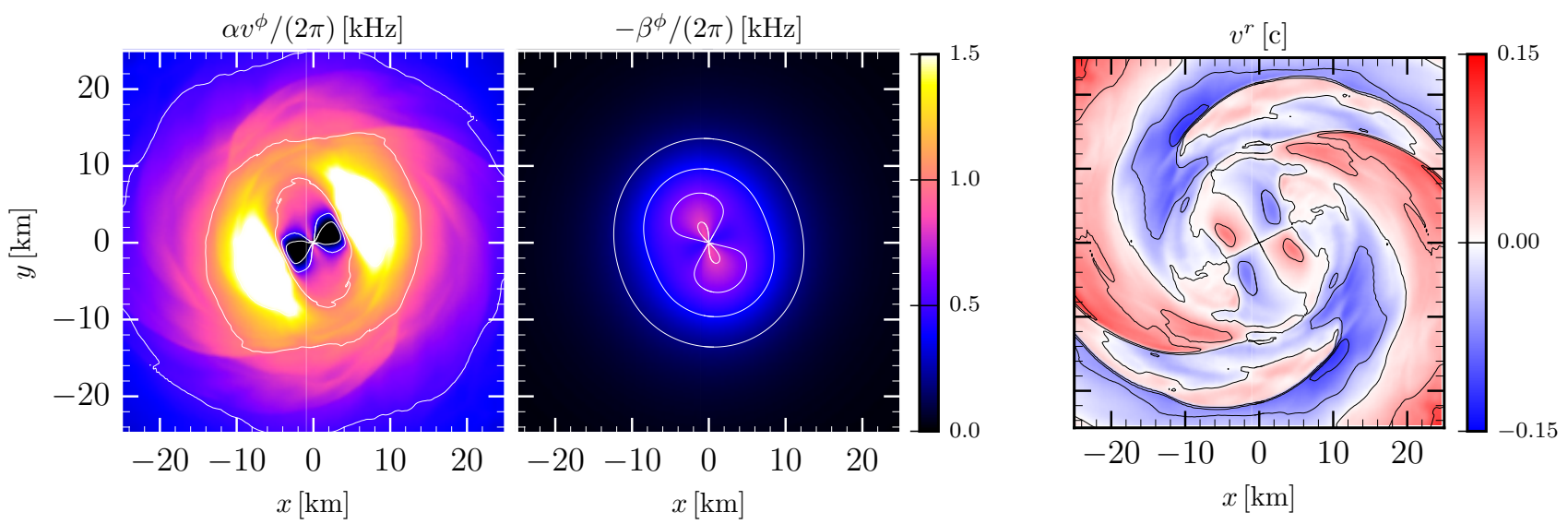

FIG. 4. Distributions on the $(x, y)$ plane for the ALF2-M135 binary at $t_{\text {III }}$ of the two contributions to the angular velocity $\Omega$. The left and central panels refer to the quantities $\alpha v^{\phi}$ and $-\beta^{\phi}$, respectively [cf. Eq. (3)]. The right panel shows instead the distribution of the radial component of the fluid three-velocity $v^{r}$.

ner regions of the HMNS, where the angular velocity can even become negative, i.e., with the minima counter-rotating relative to the outer layers of the HMNS. The largest gradients in the $\Omega$-profile take place at $\simeq 3 \mathrm{~km}$ from the center. Although both $\rho$ and $\Omega$ have marked $m=2$ distributions, it is also clear that there is an evident phase offset of $\simeq 90$ degrees between them. This feature is very robust and is present in all of the binaries we have simulated. However, to the best of our knowledge, this has not been reported before in the literature. This phase offset can be explained rather simply in terms of an extension of Bernoulli's theorem for which areas of low (pressure) rest-mass density are accompanied by regions of large velocity; this is in essence what the upper and lower panels of Fig. 3 express. A more detailed discussion of this point will be presented in Appendix A, when analysing the conservation of the Bernoulli constant associated to representative tracers.

A question that arises when investigating the spatial properties of the angular velocity $\Omega$ is whether such properties are physical and not just a gauge artefact given that $\Omega$ is, after all, a gauge-dependent quantity. Although the influence of gauge deformations has already been assessed to be small in a study performed with very similar gauges and physical conditions [20], we have performed a number of additional investigations to rule this out. Firstly, we have investigated the evolution of the relevant components of the spatial three-metric (namely, $\gamma_{r r}, \gamma_{\phi \phi}$ and $\gamma_{r \phi}$ ) and did not find a corresponding structure which could have produced the properties discussed above for the spatial distribution of $\Omega$. Secondly, we have investigated the properties of the various quantities that contribute to the angular velocity, namely, the frame dragging provided by the shift component $-\beta^{\phi}$ and the azimuthal fluid velocity $\alpha v^{\phi}$ [cf. Eq. (3)], together with the radial component of the threevelocity $v^{r}$.

Figure 4 shows the equatorial structure of these three quantities at time $t_{\mathrm{III}}$. The left panel, in particular, displays $\alpha v^{\phi}$, and has almost the same global structure as that shown by $\Omega$ (see lower right panel of Fig. 3), with the only (obvious) difference that the maximum and minimum amplitudes are smaller. The central panel of Fig. 4 shows the shift component $\beta^{\phi}$ with the same colorcode, indicating that it not only has a similar spatial structure to the rest-mass density, but it is also considerably smaller, becoming essentially zero in the outer layers of the HMNS (i.e., for $r \gtrsim 15 \mathrm{~km}$ ). Both of these facts indicate that the influence of gauge quantities on the values of $\Omega$ cannot be responsible for the 90 -degrees phase shift, which has instead a rather intuitive explanation given above.

In addition, the right panel of Fig. 4 shows the radial component of the three-velocity $v^{r}$, with red regions indicating fluid cells with outward radial motion, while blue regions refer to fluid moving inward. Note that at the outer parts of the HMNS (i.e., for $r \gtrsim 15 \mathrm{~km}$ ) the flow is mostly outwards along the two dense spiral arms. These will feed the matter ejected dynamically that will eventually lead to the production of heavy elements [84, 85]. On the other hand, the distribution of the radial velocity in the inner parts of the HMNS (i.e., for $r \lesssim 10 \mathrm{~km}$ ) shows a clear quadrupolar structure produced by the propagation of the $m=2$ rest-mass density perturbation. To clarify the properties of this structure it is sufficient to imagine an $\ell=m=2$ tidal wave moving along the surface of an otherwise spherical star. The local velocity will be a $\ell=2, m=4$ succession of positive and negative radial velocities as the tidal wave sweeps through the surface. Additional considerations along these lines will be made when discussing the motion of tracer particles in Sec. V.

In summary, all the analyses discussed so far indicate that the $m=2$ distribution of the angular velocity presented in Fig. 3 is not contaminated by gauge effects and it can be interpreted in terms of the physical manifestation of the Bernoulli theorem.

Before concluding this section we should remark that all the results presented above have been focused on the equatorial plane; however, the extensions to planes at nonzero elevation is straightforward. More specifically, we have found that the structure of the rest-mass density and rotation profiles do not 


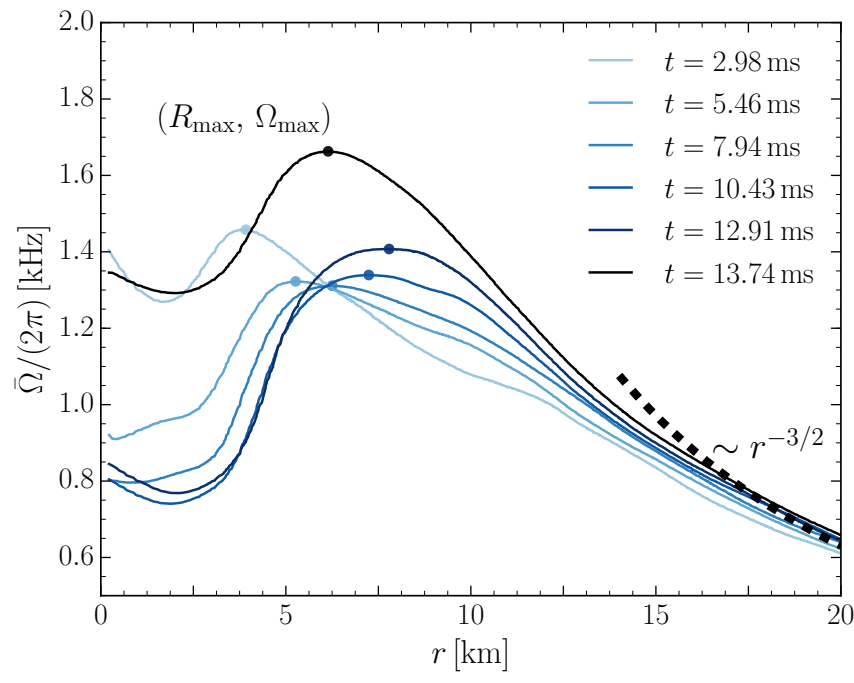

FIG. 5. Averaged fluid angular velocity $\bar{\Omega}(r) /(2 \pi) \mathrm{kHz}$ on the equatorial plane for the ALF 2-M135 binary as averaged at different times and with intervals of length $\Delta t=1 \mathrm{~ms}$. Shown as a thick dashed black line is a reference profile scaling like $r^{-3 / 2}$.

change significantly for $z<8 \mathrm{~km}$, although the $m=2$ deformation tends to be less marked. Moving further away from the equatorial plane, the angular velocity becomes almost axisymmetric, being small in the core of the HMNS, but growing to larger values in the outer layers, i.e., for $z>11 \mathrm{~km}$. At these heights, the rest-mass density has decreased considerably and the HMNS smoothly blends in with the outward-moving wind of the dynamically ejected matter and which will represent an important element of pollution of the interstellar medium [86, 87].

\section{Angular-velocity evolution: azimuthal averages}

As discussed before, for high-mass binaries the angularvelocity distribution exhibits an $m=2$ deformation that persists over long timescales (this is however not the case for low-mass binaries; see Sec. IV). Because the spacetime reaches rather quickly a stationary evolution and the deformation is progressively washed out, it is reasonable to consider azimuthal averages that, by reducing the problem to a onedimensional one, can help compare angular-velocity profiles across different EOSs. Furthermore, as we will discuss in Sec. $\mathrm{IV}$, the approximation of an azimuthal average becomes increasingly good as the mass of the system decreases and the $m=2$ deformation is more rapidly lost.

We define therefore the time- and azimuthally averaged angular velocity $\bar{\Omega}(r, t)$ as

$$
\bar{\Omega}(r, t):=\int_{t-\Delta t / 2}^{t+\Delta t / 2} \int_{-\pi}^{\pi} \Omega\left(r, \phi, t^{\prime}\right) d \phi d t^{\prime} .
$$

and show its evolution in Fig. 5 for the ALF2-M135 binary. Note that to better illustrate the time dependence, a small time averaging domain $(\Delta t=1 \mathrm{~ms})$ has been used. Six representative time segments, which span almost the whole HMNS lifetime from merger to gravitational collapse, are visualized in Fig. 5.

Soon after merger (i.e., for $t \lesssim 3 \mathrm{~ms}$ ), the angular-velocity profile varies considerably as angular momentum is transferred from the central region to the outer layers (light-shaded blue lines). As a result, the angular velocity decreases significantly in the inner regions, quickly creating a large gradient with the more rapidly rotating outer layers. The time variation of the azimuthal average is much smaller as time progresses and the HMNS reaches a stationary configuration. Blue curves with increasing shading in Fig. 5 show the rotation profiles for later time segments; clearly the qualitative global structure of all of these curves is very similar. While the inner parts $(r \lesssim 3 \mathrm{~km}$ ) of the HMNS rotate rather slowly $(\sim 1.0 \mathrm{kHz})$, a sharp increase takes place in a narrow region between $4 \mathrm{~km}$ and $5.5 \mathrm{~km}$, resulting in an absolute maximum value at radii between $7-8 \mathrm{~km}$. For larger radii, the angular velocity decreases monotonically tending to a $r^{-3 / 2}$ law for $r \gtrsim 15 \mathrm{~km}$ (see discussion in Sec. VI).

The black curve in Fig. 5 describes the rotation profile of the HMNS at the brink of collapsing to a Kerr black hole ( $t \simeq 13.7 \mathrm{~ms}$ ) and can therefore be taken as the stationary azimuthally averaged angular-velocity profile. About two milliseconds before black-hole formation, the angular-velocity profile increases and the position of the maximum moves inwards as a result of angular-momentum conservation.

\section{Temperature evolution}

Reference [20] has recently studied in great detail the temperature distribution and the fluid trajectories of a HMNS produced by a binary neutron star merger obeying the Shen, Horowitz and Teige EOS [88, 89]. Although Ref. [20] concentrates on one EOS only, their results are in very good agreement with those presented so far. We next make a closer comparison with [20] by considering the evolution of a quantity we have not yet discussed, namely, the fluid temperature. In particular, we will concentrate on the binary LS220-M132, which is the only one of our set described by an EOS with a consistent temperature, where the simulations have been performed with the Whisky THC code [68, 69].

Figure 6 shows the equatorial distributions of the temperature at $t=6.71 \mathrm{~ms}$ (left panel) and at $t=23.83 \mathrm{~ms}$ (right panel; note that the representative ALF 2-M135 binary has already collapsed at this time) when observed in a "corotating frame", that is, in a frame that is rotating at a frequency that is half of the instantaneous gravitational-wave frequency. In agreement with Ref. [20], two "hot spots" are found after the early post-merger phase (see left panel of Fig. 6) which remain stable for approximately $12 \mathrm{~ms}$. The fluid trajectories (not reported in Fig. 6 but analysed in Sec. V) indicate that the hot spots also represent vortices around which fluid elements rotate. Furthermore, the temperature distribution on the equatorial plane bears a remarkable similarity with the corresponding distribution of the angular velocity as reported in Fig. 3. In 


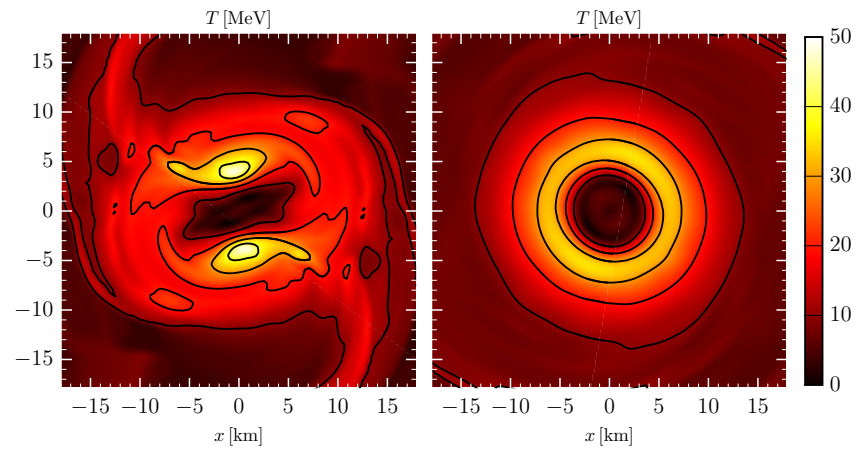

FIG. 6. Distributions on the $(x, y)$ plane and in a corotating frame of the temperature for the LS220-M132 binary at $t=6.71 \mathrm{~ms}$ (left panel) and at $t=23.83 \mathrm{~ms}$ (right panel). The isocontours correspond to $T=\{10,20,30,40,50\} \mathrm{MeV}$. Note the presence in the left panel of two hot spots, which do not coincide with the maximum rest-mass density (see also Fig. 10, which reports other quantities relative to this binary).

particular, the position of the hot spots overlaps closely with the position of the maxima in the angular-velocity distribution. This is not surprising as in these regions the fluid flow has the largest shear and compression, which are ultimately responsible for the local increase of the temperature.

Stated differently, regions of relatively smaller pressure (and rest-mass density) should also coincide with regions of larger temperature, which is what can be verified by comparing the rest-mass distribution in the left panel of Fig. 10, with the temperature in the left panel of Fig. 6, which refers to the same time. To the best of our knowledge, this is the first time this explanation is provided for the presence of the two "hot spots".

In addition to the hot spots, the temperature distribution shows local increases along the edges of the $m=2$ density perturbation (again where the fluid shear is largest in the corotating frame) and along the spiral arms, where outward moving material is ejected dynamically from the HMNS. Finally, we note that as time progresses and the HMNS reaches a stationary state, the two hot spots disappear and the temperature distribution reaches an axisymmetric pattern (see right panel of Fig. 6). Interestingly, however, the high-temperature region is not the central one, which is slowly rotating and comparatively colder, but, rather, an annular region at about 7-8 km from the center, where the (axisymmetric) angular-velocity distribution varies more rapidly.

Although the discussion in this section has mainly focussed on the ALF2-M135 and LS220-M132 binaries, the results are qualitatively similar for all of the high-mass binaries of our sample. Of course, small quantitative differences in terms of the maximum angular velocity, or the precise position of the maxima of the $m=2$ deformations in $\rho$ and $\Omega$ will depend on the stiffness of the EOS, but these still obey the overall behaviour discussed so far; a more detailed comparison of the angular-velocity profiles across the various EOSs is presented in Sec. VI.

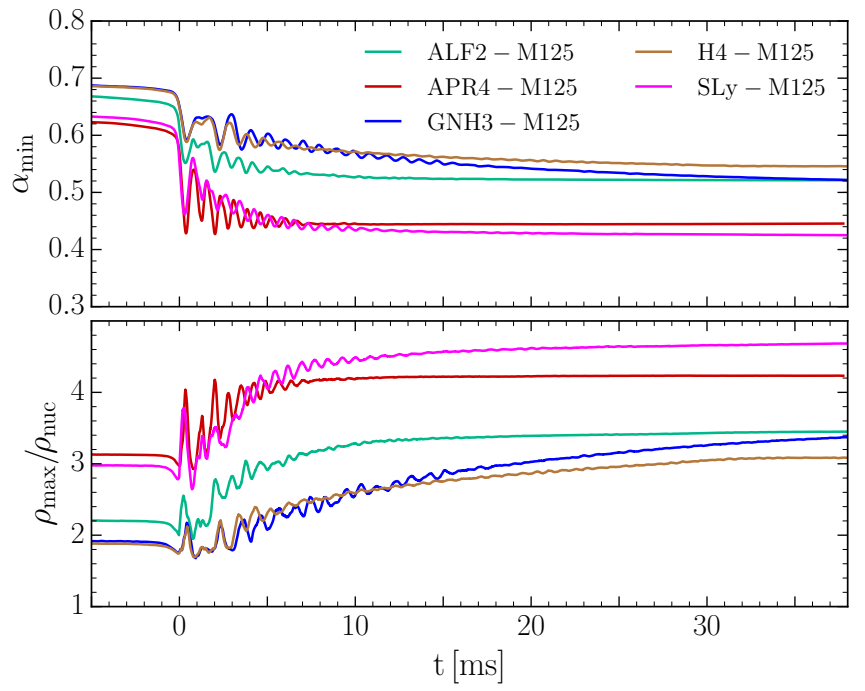

FIG. 7. Minimum value of the lapse function $\alpha_{\min }$ (upper panel) and maximum of the rest-mass density $\rho_{\max }$ in units of $\rho_{\text {nuc }}$ (lower panel) versus time in milliseconds after the merger for the low-mass simulations; this figure should be contrasted with Fig. 1.

\section{LOW-MASS BINARIES}

We turn now our attention to low-mass binaries. For this case we take the ALF2 EOS as a representative EOS and concentrate on an equal-mass binary with total mass $2 \times 1.25 M_{\odot}$, i.e., ALF 2-M125. In analogy with Fig. 1, we show in Fig. 7 the evolution of the minimum value of the lapse function and of the maximum rest-mass density for our five EOSs. None of the resulting HMNS collapses to a black hole within the simulated time range because of the smaller initial mass of the binaries (they will likely collapse on timescales of $\sim 100 \mathrm{~ms}$; see [13] and the discussion in Appendix of Ref. [48]). It is also interesting to note that the post-merger oscillations in the maximum rest-mass density are all suppressed within about 10-15 ms from the merger, with stiffer EOSs (e.g., GNH3) requiring more time than the softer ones (e.g., APR4).

Similarly, we display in Fig. 8 the emitted gravitational waves of the late inspiral, merger and post-merger, which should be contrasted with the corresponding emission for the high-mass binary shown in Fig. 2. Note that the gravitationalwave amplitude peak at the merger is comparable with that of the high-mass run, but also that within the first $10 \mathrm{~ms}$ after merger it decreases considerably to become only about $20 \%$ of that in the late inspiral. This is due to the rapid disappearance of the non-axisymmetric deformation of the rest-mass density in the HMNS, which attains an almost axisymmetric distribution within $\simeq 20 \mathrm{~ms}$ after the merger (see upper panels in Fig. 9). The three different times indicated in Fig. 8 refer to $t=1 / 4,1 / 2$ and $t=3 t_{\text {fin }} / 4$, where $t_{\text {fin }}=40.38 \mathrm{~ms}$ is the time when the simulation is terminated.

Figure 9 reports the distributions on the equatorial plane of the rest-mass density (upper panels) and of the angular velocity (lower panels) at the three different times indicated in Fig. 
8. This figure should be compared with Fig. 3, which refers to a high-mass binary of the same (ALF2) EOS. Note that for $t \lesssim 16 \mathrm{~ms}$, the rotation profile (see lower left panel in Fig. 9) shows the same qualitative structure as for the high-mass case, even though the overall values of $\Omega$ are somewhat lower (note the different color scale in Figs. 3 and 9). Although in a weaker form, the low-mass binary also shows the 90-degree shift between the $m=2$ deformation in the rest-mass density and in the angular velocity, which we have discussed in Sec. III B in terms of the manifestation of the Bernoulli theorem [cf. Eq. (A1)]. Furthermore, for $t \gtrsim 16 \mathrm{~ms}$ (see middle and right panels in Fig. 9) the rest-mass density and the rotation profile have reached a stationary state in which a small $m=2$ perturbation is still present, but is subdominant when compared to the overall axial symmetry. The inner part of the HMNS $(r \leq 6 \mathrm{~km})$ is where the rest-mass density is the largest, but is also rotating rather slowly $(\Omega \simeq 0.5 \mathrm{kHz})$; this region is much broader than in the high-mass binary and the sharp transition to a $r^{-3 / 2}$ outer profile takes place at a larger radius $(6.5 \lesssim r \lesssim 8.5 \mathrm{~km})$. The panels on the right column of Fig. 9 clearly indicate that at later times the HMNS has reached a high degree of axial symmetry, although not a complete one, since gravitational waves are still being emitted (cf. Fig. 8).

As discussed in Sec. III for the high-mass binaries, here too we can comment that the analysis carried out on the equatorial plane remains qualitatively valid also at nonzero elevations, with the rest-mass density and rotation profiles not varying significantly for $z \lesssim 9 \mathrm{~km}$, and maintaining the overall axisymmetry. Furthermore, as the HMNS reaches rest-mass densities that are comparable with that of the outgoing wind, for $z \gtrsim 10 \mathrm{~km}$, the angular velocity increases in the central regions, where it has the largest values.

Finally, we conclude this section remarking that much of the properties discussed so far for the ALF 2-M125 binary remain qualitatively true for all of the low-mass binaries considered in our sample of models. Once again, small quantitative differences do appear when considering the maximum angular velocity, or the precise position of the maxima of the $m=2$ deformations in $\rho$ and $\Omega$, which obviously depend on the stiffness of the EOS. However, the overall behaviour discussed so far can be taken to be representative for binaries with these masses and a large class of EOSs.

\section{TRACER PARTICLES EVOLUTION}

To further strengthen the conclusions reached above about the physical significance of the properties of the angularvelocity distribution discussed in the previous section, we present below a complementary analysis making use of the flowlines tracked by massless tracer particles that are advected in the flow (this is not the first time tracer particles are used in fully general-relativistic simulations and recent related work can be found in Refs. [20, 90]). More specifically, we concentrate on the binary LS220-M132, as evolved with the WhiskyTHC code $[68,69]$. Details on our implementation of tracers can be found in [90], while subtleties of the infor-

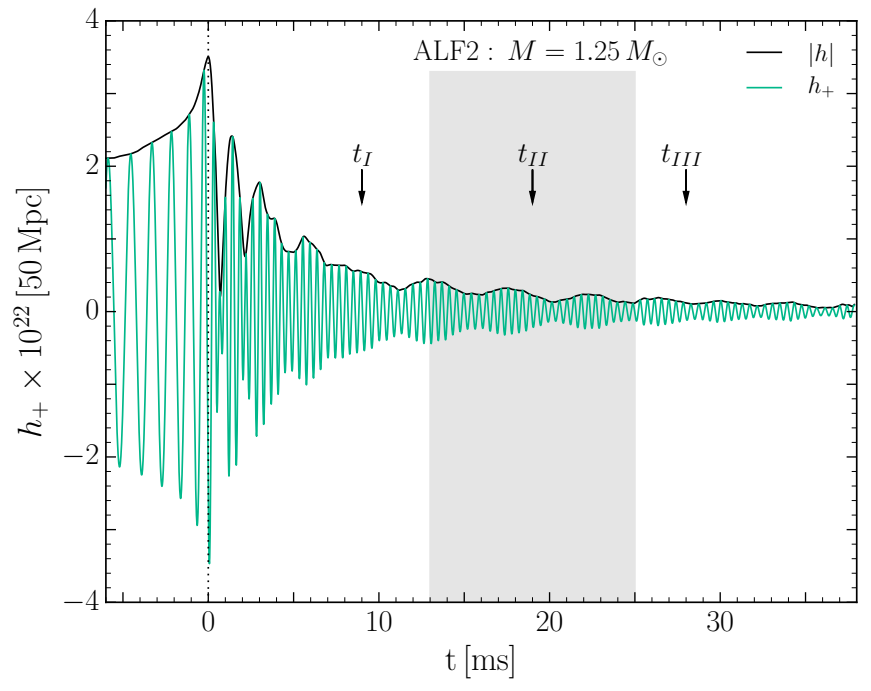

FIG. 8. Gravitational-wave amplitude $|h|$ (black line) and strain amplitude in the + polarisation $h_{+}$(green line) for the ALF2-M125 binary at a distance of $50 \mathrm{Mpc}$. Shaded in gray is the portion where a time average is performed, while the arrows indicate the times when representative distributions of the rest-mass density and angular velocity are shown in Fig. 9. Finally, the dotted vertical line marks the time of merger. This figure should be contrasted with the equivalent one, Fig. 2, for the high-mass binaries.

mation that can be derived from them will be presented in a related work [91].

The upper panels of Fig. 10 report, for the LS220-M132 binary and at time $t=6.7 \mathrm{~ms}$, the rest-mass density $\rho$ (left panel), the angular velocity $\Omega$ in the corotating frame (middle panel) and in the Eulerian frame (right panel). Similarly, the bottom row of panels in Fig. 10 shows the same quantities, but for a later time of $t=23.8 \mathrm{~ms}$. The times shown are representative ones but the dynamics for this EOS is qualitatively very similar to those presented in Secs. III and IV. The only difference is that although the binary LS220-M132 belongs to the high-mass class, its evolution does not lead to a collapse to a black hole over the timescale during which the simulations have been carried out, i.e., $\sim 27.3 \mathrm{~ms}$ after merger.

Also shown in Fig. 10 are the flowlines of several tracer particles that remain close to the $(x, y)$ plane (i.e., with small velocity in the $z$ direction) and for which we introduce a novel visualization technique. More specifically, we show only the final part of the flowlines (i.e., for the last $\simeq 0.285 \mathrm{~ms}$ ), using small dots to indicate the particle position at the time indicated in the frame. Furthermore, the initial parts of the trajectories have increasing transparency so as to highlight the final part of the trajectories. This approach has at least two advantages. First, it provides a measure of the linear velocity (faster tracers leave longer tracks); second, the presence of the filled dots and the increasing transparency allow one to read-off the direction of motion.

The top panels in Fig. 10 show the dynamics of the fluid in the inner parts of the HMNS and highlight that two distinct regions can be identified. The first region is in the core 

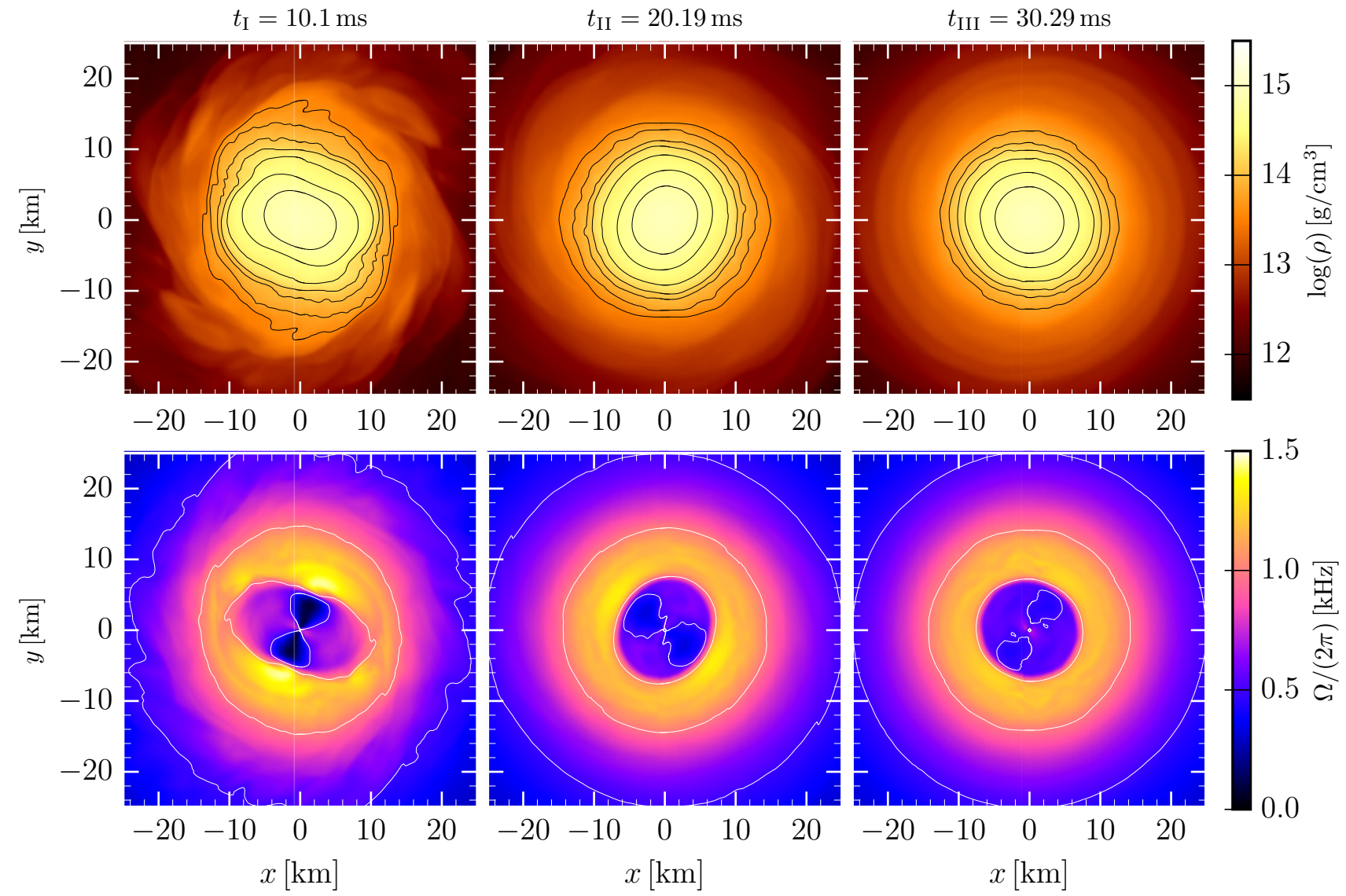

FIG. 9. Distributions of the rest-mass density (upper row, log scale) and of the fluid angular velocity (lower row) in the $(x, y)$ plane for the ALF 2-M125 binary at three different post-merger times as indicated in Fig. 8. The isocontours have been $\operatorname{drawn}$ at $\log (\rho)=13.6+0.2 n$ (upper row) and $\Omega=\{0,0.5,1.0,1.5,2.0\} \mathrm{kHz}$ (lower row), $n \in \mathbb{N}$.

of the HMNS, where there is an ellipsoidal structure orthogonal to the angular-velocity distribution. Within this ellipsoid, fluid elements essentially move clockwise along isobaric surfaces, with linear velocities that are rather small in the inner regions. In addition to this ellipsoidal motion, the tracers also show the presence of two small "vortices", i.e., regions of increased vorticity in this frame, which also coincide with the regions of highest angular velocity, and which border the areas where velocity drops almost to zero (in this frame). Note that it appears that the tracers that are "trapped" in these vortices where they remain without traversing the boundary to the central ellipsoid and where they have an inverse sense of rotation (counter-clockwise). Stated differently, the vorticity distribution in the corotating frame would show two islands of vorticity with different signs, referring to clock and counterclock wise rotation. Tracers in one region do not migrate to the other region. This is mostly the result of using a corotating frame in a flow that is differentially rotating. It is quite intuitive, in fact, that if $\Omega$ is positive but not uniform, the transformation to a corotating frame amounts to a net subtraction of a positive amount of angular velocity, hence leading to areas of now negative angular velocity. This becomes more apparent when considering the corresponding picture in the Eulerian frame, as shown in the right panels of Fig. 10.

The bottom panels of Fig. 10, on the other hand, refer to a much later stage of the HMNS evolution (i.e., $t=23.83 \mathrm{~ms}$ ) and clearly show that by this time the HMNS has attained an almost axisymmetric structure, combined with a much smaller $m=2$ perturbation. As discussed in the previous cases, also here the rotational profile of the HMNS contains an inner area with $r \lesssim 5 \mathrm{~km}$ which is rotating slowly and almost uniformly at $\Omega \approx 0.5 \mathrm{kHz}$, followed by a sharp increase at $5 \lesssim r \lesssim 7 \mathrm{~km}$ reaching a maximum value $\Omega \approx 1.5 \mathrm{kHz}$ at $r \approx 8 \mathrm{~km}$ and decreasing continuously for $r \gtrsim 8 \mathrm{~km}^{2}$. Since this behaviour follows the one described previously for hybrid EOSs, it suggests that both the rest-mass density and the angular-velocity distributions are preserved when using a fully three-dimensional EOS and radiative losses are taken into ac-

\footnotetext{
2 Note that the angular-velocity distribution in the lower central panel of Fig. 10 refers to the corotating frame and that this frame is rotating at half the angular frequency of the emitted gravitational waves, $\Omega_{\mathrm{GW}}$. Because the maximum of the angular velocity $\Omega_{\mathrm{max}}$ is of the order of $\Omega_{\mathrm{GW}} / 2$ (cf. left panel of Fig. 13), the ring structure in this panel is approximately at zero angular velocity.
} 

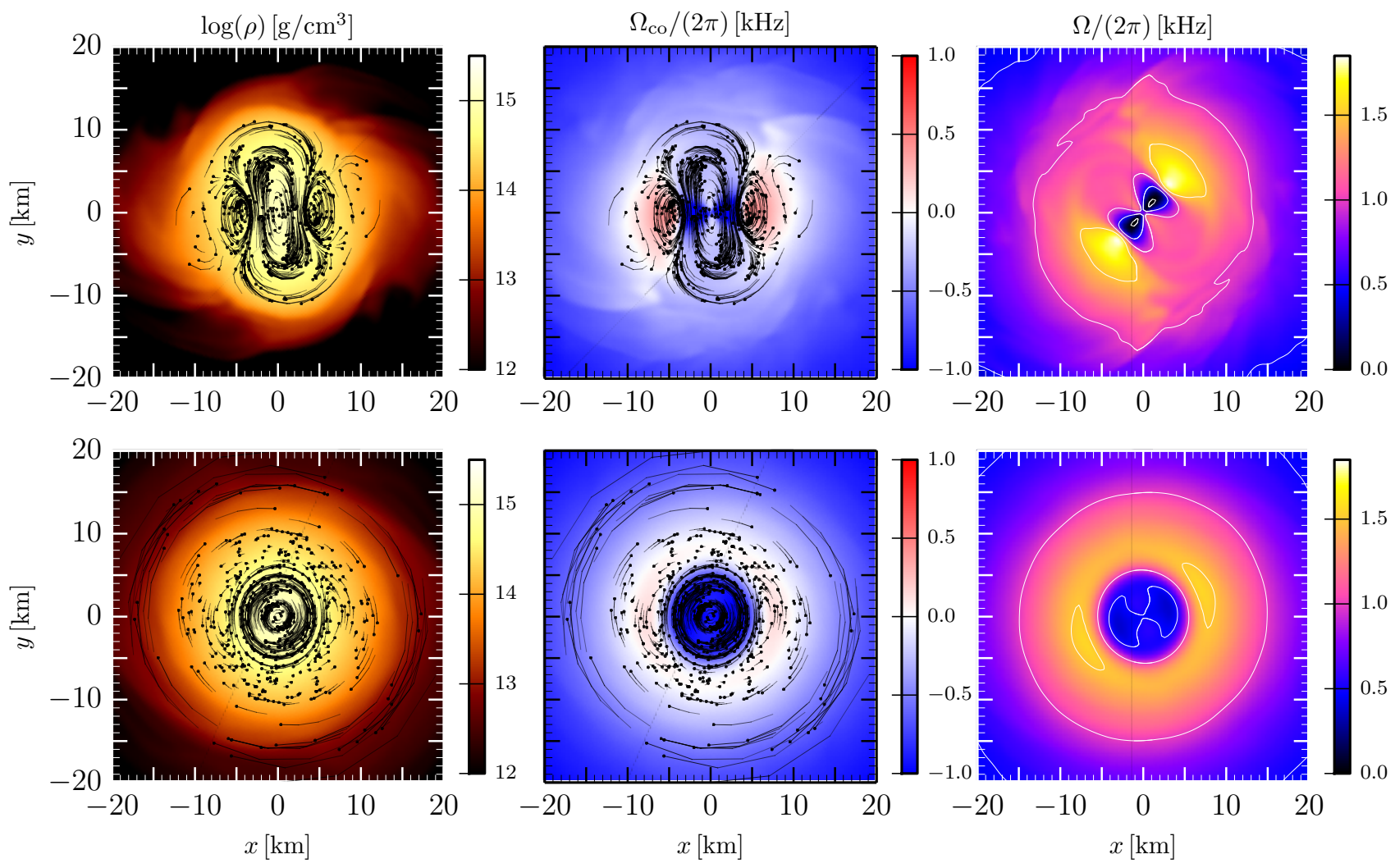

FIG. 10. Distributions on the $(x, y)$ plane and in the corotating frame of the rest-mass density (left panels), and of the angular velocity (middle panels) for the LS220-M132 binary, where $\Omega_{\mathrm{co}}:=\Omega-\Omega_{\mathrm{f}}$ where $\Omega_{\mathrm{f}}=1.368 \mathrm{kHz}$ (top), $1.425 \mathrm{kHz}$ (bottom). Shown instead in the right panels is the distribution of the angular velocity, $\Omega$, in the Eulerian frame. The top row refers to $t=6.71 \mathrm{~ms}$, while the bottom one to $t=23.83 \mathrm{~ms}$. Also shown are portions of the flowlines of several tracer particles that remain close to the $(x, y)$ plane and for which we show only the final part of the flowlines (i.e., for the last $\simeq 0.285 \mathrm{~ms}$ ), using small dots to indicate the particle position at the time indicated in the frame. In addition, the initial parts of the trajectories have increasing transparency so as to highlight the final part of the trajectories.

count. In turn, this confirms that an analysis carried out with piecewise polytropes and a thermal component does not introduce a bias in the results.

The tracers in the lower panels of Fig. 10 further illustrate the axisymmetric nature of the flow, with the fluid moving along essentially circular orbits that are tangent to isobaric surfaces. The quasi-circularity is shown in the spacetime diagram reported in Fig. 11, where we show the worldlines of selected tracers in the relevant region of the HMNS and after passing them through a running-average window of $5 \mathrm{~ms}$ to remove the high-frequency jitter. Note that after the transient period, where angular momentum is transferred out and particles move to lower rest-mass density regions, the tracers remain at essentially constant radial coordinates.

It is interesting to note that because the angular velocity refers to the corotating frame and because this frame is rotating at half the gravitational-wave frequency $\left(\Omega_{\mathrm{GW}} / 2 \approx\right.$ $\Omega_{2} / 2 \approx \Omega_{\max }$, see Fig. 13 of Section VI), tracers in the inner and outer regions of the HMNS will be both rotating clockwise while tracers belonging to the intermediate regions are almost at rest (white regions). Only those tracers that are trapped in the vortices are moving counter-clockwise (light red regions).

To recap, the analysis of the motion of tracer particles discussed in this section indicates that the angular-velocity distributions presented so far are not contaminated by gauge effects, but rather reflect physically meaningful quantities. The angular-velocity $\Omega$ is defined directly in terms of the lapse and shift, which are gauge-dependent quantities and could potentially change the behaviour of the angular-velocity depending on the gauge selected. The evolution of the tracers are comoving with the fluid elements [91] and thus they follow the fluid evolution. As shown, they exhibit behaviour as predicted by the gauge-dependent quantities and illustrate the robustness of our results to the choice of gauge. An additional use of the tracers, explained in detail in Appendix A, will further illustrate the origin of the phase offset demonstrated in Fig. 3. 


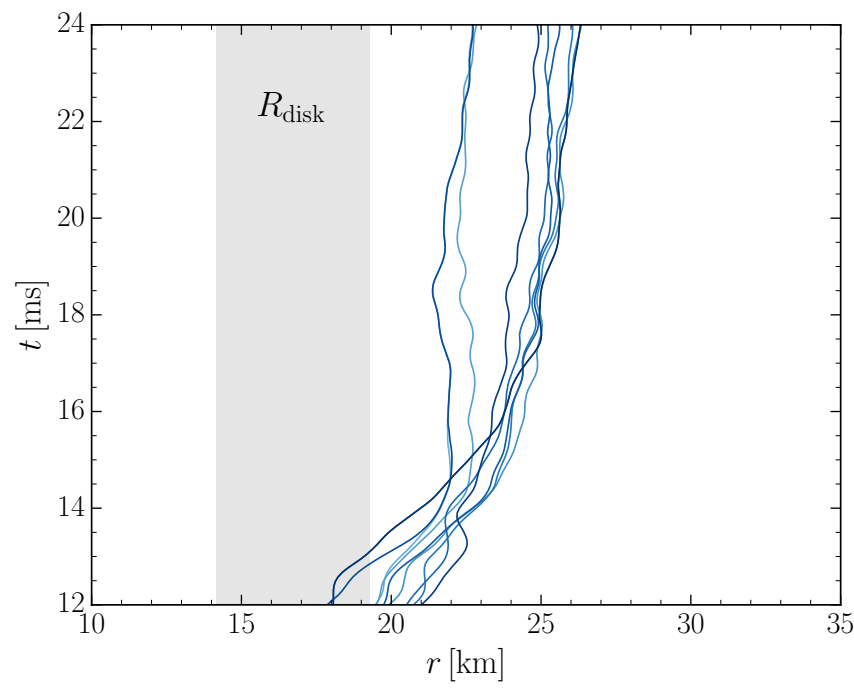

FIG. 11. Worldlines of selected tracers in the outer regions of the HMNS where the angular frequencies scale like $r^{-3 / 2}$. Note how in these regions the tracers remain at essentially constant radial coordinates; the gray-shaded area shows the region where $\Omega(r) \propto r^{-3 / 2}$ (see Sec. VI A for a definition and discussion).

\section{VI. “QUASI-UNIVERSAL” BEHAVIOUR}

\section{A. Averaged profiles}

A particularly interesting result of our analysis emerges when comparing the time- and azimuthally averaged profiles of the angular velocity across the various EOSs and masses. We recall that the averaging procedure has been discussed in Sec. III C and requires a proper choice of the integration interval, which is different in the case of high-mass binaries (where the HMNS collapses to a black hole) and that of lowmass binaries (where the HMNS survives through all the simulated time). To maintain a certain consistency across these two cases, we have performed the time averages across a time interval $\Delta t$ [see Eq. (9)] centered around $t_{\text {fin }} / 2$ and with extent $t_{\text {fin }} / 3$, where $t_{\text {fin }}$ corresponds either to the time of blackhole formation (in the case of high-mass binaries) or to the final time of the simulation (in the case of low-mass binaries). These time intervals have been indicated with gray-shaded regions in Figs. 2 and 8, respectively (see Appendix B for a discussion on the impact of resolution on the lifetime of the HMNS and on the averages).

Figure 12 shows the results of the time- and azimuthally averaged angular-velocity profiles as described above and as computed for the high and low-mass simulations with different EOSs. Besides being in good agreement with the results of previous works where a much smaller number of EOSs was investigated $[19,20]$, the overall behaviour of the angularvelocity profiles shown in Fig. 12 hints at a behaviour that is only weakly dependent on the EOS and could therefore be considered "quasi-universal".

More specifically, all profiles show the presence of a slowly

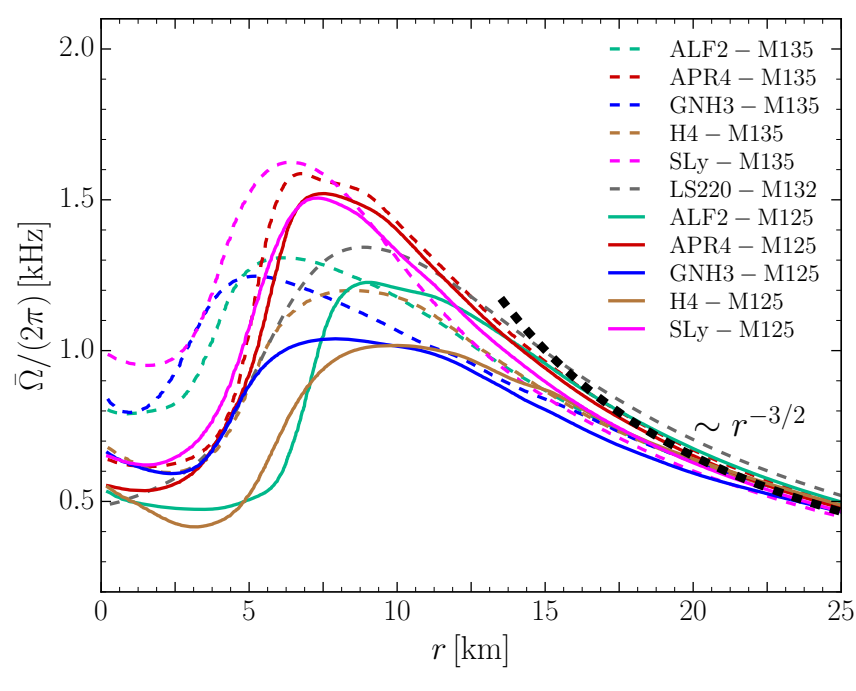

FIG. 12. Comparison of the time- and azimuthally averaged rotation profiles for different EOSs. Solid curves show the profiles for highmass runs $\left(M=1.35 M_{\odot}\right)$, whereas dashed curves refer to low-mass simulations $\left(M=1.25 M_{\odot}\right)$. Shown as a thick dashed black line is a reference profile scaling like $r^{-3 / 2}$.

and essentially uniformly rotating inner core, which is then joined by a rapidly rotating outer region, hence decreasing outwards as $r^{-3 / 2}$, and where the rest-mass density is that typical of neutron-star crusts (see also Fig. 14 and the discussion below). The transition between the slow inner core and the rapidly rotating exterior takes place across a narrow region which is only $3-4 \mathrm{~km}$ wide. It is across this layer that shear forces are the largest and consequently local temperature increases are present (see Fig. 6). This differential rotation profile is rather different from the one normally considered in the literature, i.e., the $j$ - constant law that has been explored in the past both in equilibrium configurations [3-8] and in dynamical ones [92-102].

Although very robust, small differences do appear within this "quasi-universal" behaviour. In particular, the spatial size of the slow inner core depends both on the EOS and on the initial mass of the binary, with smaller-mass binaries having in general larger slower cores; as an example, for the highmass run of the GNH3 and SLy EOS the slowly rotating core is rather small ( $r \lesssim 4 \mathrm{~km}$ ) while for the low-mass run of the ALF2 EOS it extends up to $r \lesssim 7 \mathrm{~km}$. In addition, the maximum angular velocity attained in the outer region also depends weakly on the EOS, being naturally higher for soft EOSs and smaller for stiff EOSs.

Inspired by the approach suggested in Refs. [30, 36, 6466], we next discuss how to relate the "quasi-universal" features of the averaged angular-velocity profiles with some of the properties of the merging neutron stars, such as the mass and radius, when they are at infinite separation. Such correlations are summarised in Fig. 13. We start by considering how the maximum angular velocity $\Omega_{\max }$ of the averaged profiles relates to the angular frequency corresponding to the largest peaks of the post-merger power spectral density 


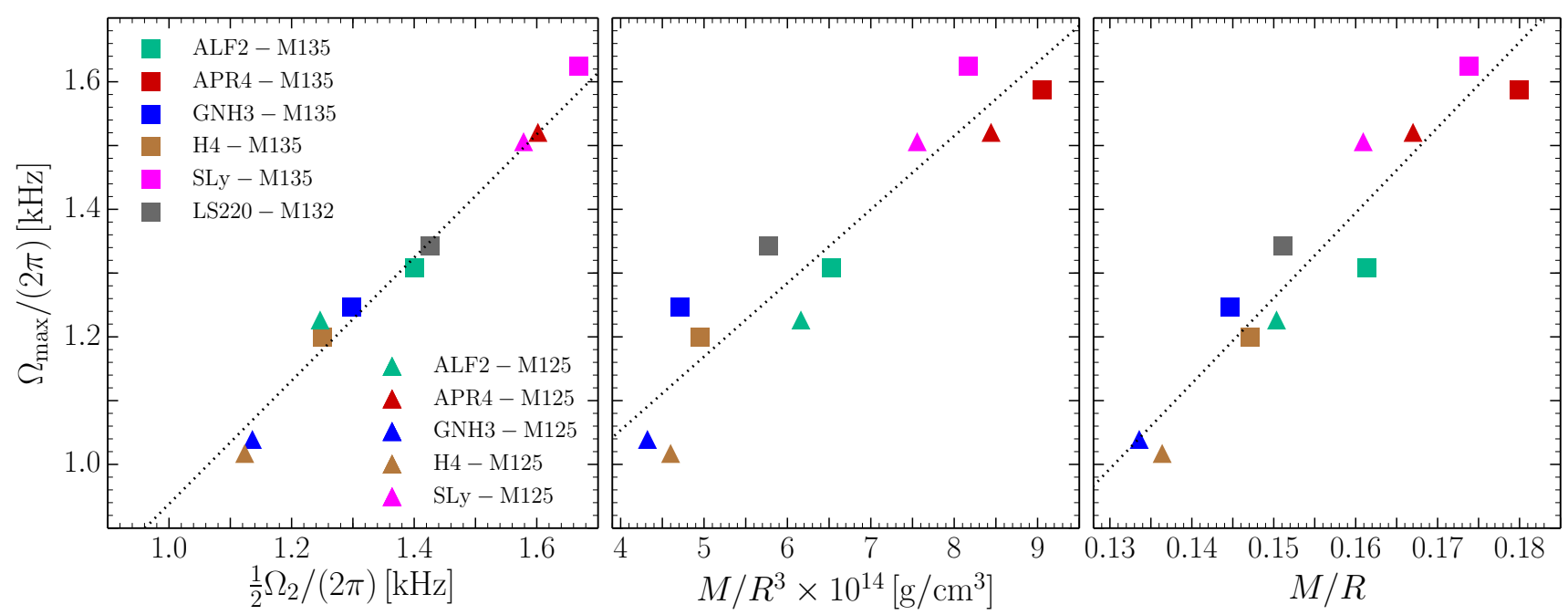

FIG. 13. Left panel: maximum value $\Omega_{\max }$ of the time- and azimuthally averaged rotation profiles (see Fig. 12) as a function of (half of) the main gravitational-wave frequency of the emitted by the HMNS $\Omega_{2}$. Middle panel: $\Omega_{\max }$ as a function of the average rest-mass density $\bar{\rho}:=M / R^{3}$ relative to the initial stellar models. Right panel: $\Omega_{\max }$ as a function of the initial stellar compactness $\mathcal{C}:=M / R$.

$\Omega_{2}:=2 \pi f_{2}$ (left panel of Fig. 13; see $[30,36,66]$ for a definition and discussion of the various frequencies of the postmerger signal). This frequency is customarily interpreted as twice the spinning frequency of the $m=2$-deformed HMNS $[66,103]$ and is therefore not surprising that it should tightly correlate with the maximum angular frequency of the averaged angular-velocity profiles. What is less obvious is that the value of $\Omega_{\max }$ reported in Fig. 13 does not change significantly with time and is therefore also the value of $\Omega_{\max }$ at the end of the simulations, when the $m=2$ deformation has either been washed out or is small. Stated differently, when the HMNS has reached an almost axisymmetric configuration, the gravitational-wave frequency $\Omega_{\mathrm{GW}} \simeq \Omega_{2}$ can still be used to measure the maximum angular velocity of the fluid. In addition, it is worth remarking that the correlation shown between $\Omega_{\max }$ and $\Omega_{2}$ provides an additional confirmation that the measurements of the angular-velocity distributions discussed in the previous section (see discussion in Sec. III) is physically meaningful. While in fact $\Omega_{\max }$ is gauge dependent, $\Omega_{2}$ is one of the few gauge-independent quantities of our simulations.

Shown instead in the central and right panels of Fig. 13 are the correlations of $\Omega_{\max }$ with the average rest-mass density $\bar{\rho}:=M / R^{3}$ and the stellar compactness $\mathcal{C}:=M / R$. Also in this case, an essentially linear correlation exists, which is easy to explain. Stiffer EOSs will lead to HMNSs that have comparatively larger radii and hence smaller average densities; in turn, smaller angular velocities will be necessary to attain a quasi-stationary hydrostatic equilibrium for the HMNS. Furthermore, for a given EOS, low-mass binaries will have comparatively smaller average densities, thus explaining why, for the same EOS, binaries reported with triangles have systematically lower averaged maximum angular velocities (cf. central panel of Fig. 13). Finally, the line of arguments described above for the average densities applies unmodified also for the stellar compactness (cf. right panel of Fig. 13).

We have already commented, when discussing Fig. 12, that the fluid flow in the outer regions of the HMNS, i.e., for $r \gtrsim 15 \mathrm{~km}$, exhibits a profile scaling like $r^{-3 / 2}$. To substantiate this claim we recall that if, at the time the HMNS has reached a quasi-stationary configuration, we assume the spacetime at sufficiently large radii to be sufficiently close to that of a Kerr black hole, so that the geodesic motion of fluid elements on the equatorial plane will have orbital angular frequencies given by Kepler's expression [50]

$$
\Omega_{\mathrm{Kep}}(r)=\frac{\sqrt{M}}{\sqrt{r^{3}}+a \sqrt{M}},
$$

where $a:=J / M$ is the spin parameter of the Kerr black hole, $J$ is the total angular momentum, and $M$ is the total gravitational mass. Of course this approximation is very crude since the HMNS's spacetime is not that of a Kerr black hole, indeed the low-mass models do not collapse, and the fluid motion is not entirely geodetic, but it is also true that this approximation is very helpful to characterise the fluid motions in the outer regions. We can therefore introduce the quantity

$$
\chi(r):=\Omega^{2}(r) r^{3},
$$

which would tend asymptotically to the mass of the black hole if $\Omega=\Omega_{\mathrm{Kep}}$, if the motion was a geodetic one, and if this was a Kerr spacetime; obviously, none of these conditions are actually met here. However, as we discuss below, we can use the radial dependence of this quantity to measure where the flow starts having angular frequencies scaling like $r^{-3 / 2}$.

This is shown in Fig. 14, which reports the behaviour of $\chi(r)$ for the binaries simulated. Clearly, all of the profiles converge to a rather constant value for large radii, thus indicating that indeed the low rest-mass density regions of the HMNS exhibit an a flow with angular frequencies scaling like $r^{-3 / 2}$. 


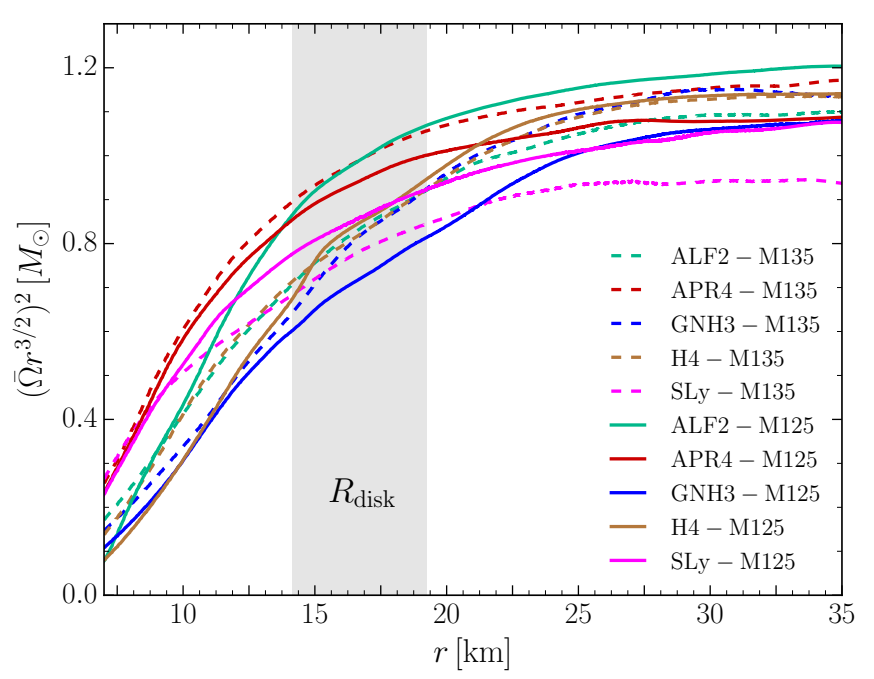

FIG. 14. Radial dependence of the quantity $\chi(r):=\bar{\Omega}^{2} r^{3}$ for all of the simulated binaries. Note that all profiles reach an almost constant value for $r \gtrsim 25 \mathrm{~km}$. The gray-shaded area shows the region where the flow starts to having angular frequencies scaling like $r^{-3 / 2}$; see Table II for the exact values of $R_{\text {disk }}$ for the various EOSs.

In order to determine where this happens, we follow a very phenomenological approach and compute the scale height of $\chi$, that is, $\chi^{\prime} / \chi:=(d \chi / d r) / \chi$. Since $\chi \rightarrow$ const. for a flow with $\Omega(r) \propto r^{-3 / 2}, \chi^{\prime} / \chi$ will be zero when this happens. Of course, $\chi \rightarrow$ const. only asymptotically and so we approximate the location of the transition to a disk as the place where the monotonically decreasing function $\chi^{\prime} / \chi$ reaches a sufficiently small value, which we here choose to be $\chi^{\prime} / \chi \leq 0.05$.

The importance of having a disk in the outer regions of the HMNS is at least twofold. First, since a flow with $\Omega \propto r^{-3 / 2}$ satisfies the Rayleigh criterion of rotating fluids against axisymmetric perturbations ${ }^{3}$, the differentially rotating "disk" surrounding the HMNS will probably accrete onto the uniformly rotating core of the HMNS only on a dissipative timescale. Therefore, it is possible that it will not affect the long-term stability required in the proto-magnetar model for short gamma-ray bursts [31-33] and the subsequent extended X-ray emission [34]. Second, once the core of the HMNS eventually collapses to a rotating black hole, the presence of a certain amount of mass on stable orbits will guarantee that the black hole will not be "naked", as suggested in Ref. [104], but rather surrounded by a torus, which would then lead to the potential formation of a relativistic jet. We will discuss this point in more detail in the following section.

\footnotetext{
${ }^{3}$ We recall that this classical-physics criterion can also be seen as requiring that the specific angular momentum $j=\Omega(r) r^{2}$ increases outwards for a stably rotating fluid configuration.
}

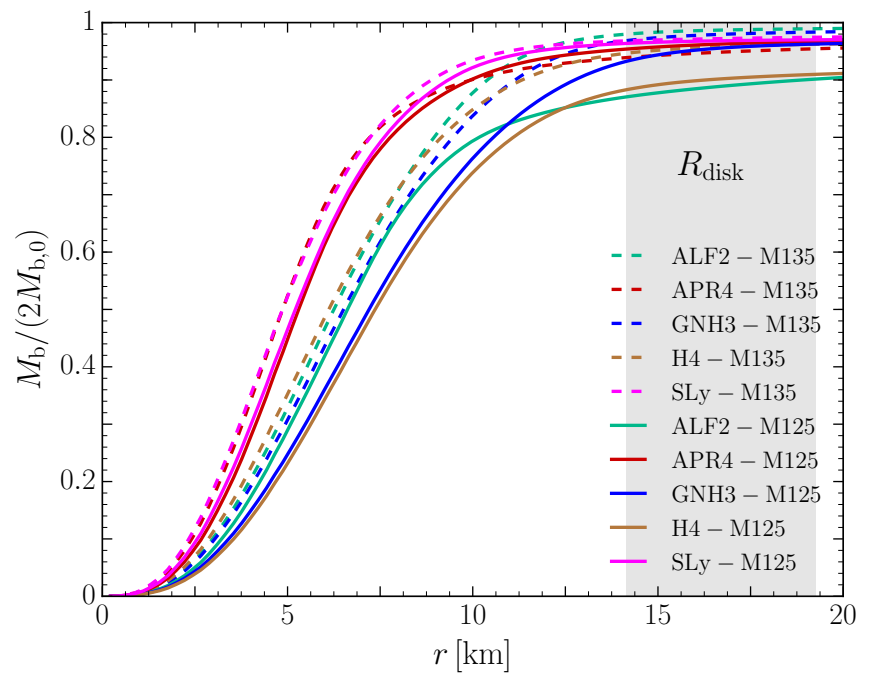

FIG. 15. Integrated rest mass $M_{\mathrm{b}}(r)$ as function of the radial coordinate and normalised with the total initial rest mass. The gray shaded area shows the region where the disk starts; see Table II for the exact values of $R_{\text {disk }}$ for the various EOSs.

\section{B. Mass in the "disk"}

The distribution of rest mass in the HMNS is of great astrophysical importance as it regulates the amount of mass that is ejected in the merger and that can subsequently feed $r$ process nucleosynthesis and an electromagnetic counterpart to the merger via the radioactive decay of by-products of the r-process (i.e., via a macronova) (see, e.g., [85, 105-114]). In addition, and as mentioned in the previous section, the knowledge of the rest-mass distribution in the HMNS, and in particular of the portion of it in the disk, is important to determine how much of the HMNS will "survive" the process of gravitational collapse of the HMNS to a black hole and end up in building a torus around the black hole.

As a result, we have computed the rest-mass distribution as a function of the radial distance from the origin as

$$
M_{\mathrm{b}}(\tilde{r}):=\int_{0}^{2 \pi} \int_{0}^{\pi} \int_{0}^{\tilde{r}} \sqrt{\gamma} W \rho r^{2} \sin (\theta) d r d \theta d \phi,
$$

with $W=\alpha u^{t}$ being the Lorentz factor. Figure 15 illustrates the radial dependence of the total rest mass in the HMNSs when normalised with the total initial rest mass $2 M_{\mathrm{b}, 0}$. Note that both for the high- and low-mass binaries the distributions refer to times which are in the middle of the averaging intervals in Figs. 2 and 8 (i.e., at $t=t_{\mathrm{BH}} / 2$ for the high-mass binaries and at $t=t_{\text {fin }} / 2$ for the low-mass binaries).

Irrespective of the EOS and initial mass, all of the restmass distributions indicate that the rest mass $M_{\mathrm{b}}(r)$ does not change significantly for $r \gtrsim 15 \mathrm{~km}$, so that the missing amount of rest mass is the one that has been ejected dynamically soon after the merger; note that largest majority of this ejected matter is gravitationally bound and only a very small fraction of it will be ejected and unbound [84, 85]. Figure 
15 also shows a somewhat expected result, namely, that binaries with softer EOSs (e.g., APR4 or SLy) have considerably more compact rest-mass distributions, reaching $80 \%$ of the total within only $r \lesssim 7.5 \mathrm{~km}$, quite independently of the initial mass of the binary. By contrast, binaries with stiffer EOSs (e.g., GNH3 or H4) have less compact distributions, reaching $80 \%$ of the total only for $r \lesssim 10 \mathrm{~km}$, and a bit less for lowmass binaries.

Another useful measure of the rest-mass distribution is that of the mass in the "disk", which we define to be the rest mass confined in the region of the HMNS in the disk. More specifically, if $R_{\text {disk }}$ is the radial location (on the equatorial plane) where the disk starts (i.e., where $\chi^{\prime} / \chi \leq 0.05$ ), then we define the rest mass in the disk as $M_{\mathrm{b}, \text { disk }}:=2 M_{\mathrm{b}, 0}-M_{\mathrm{b}}\left(R_{\mathrm{disk}}\right)$. Another interesting notion of mass is that that is inside the maximum value of the averaged angular-velocity profile. After defining $R_{\Omega_{\max }}$ as the location where the angular-velocity profile reaches its maximum, and recalling that i.e., $R_{\Omega_{\max }}<$ $R_{\text {disk }}$, we can calculate the mass inside $R_{\Omega_{\max }}$ using the same expression (12); we refer to this mass as to $M_{b, \Omega_{\max }}$. The masses in the disk surrounding the quasi-uniformly rotating inner core of the HMNS and that outside the angular-velocity maximum are summarised in Table II for all of the binaries simulated. Overall, they indicate that the mass outside $R_{\Omega_{\max }}$ and the mass outside $R_{\text {disk }}$ are rather similar (the former being slightly larger since since $\left.R_{\Omega_{\max }} \lesssim R_{\text {disk }}\right)$ and can be quite large, being almost $0.3 M_{\odot}$ for some of the soft-EOS low-mass binaries and of the order of $0.1 M_{\odot}$ for the other binaries. Long-term angular-momentum transport and neutrino radiation will change quantitatively these values, but we do not expect them to change the qualitative picture that lowmass soft-EOS binaries will have comparatively larger disks.

\section{Influence of the thermal component}

The final section of this paper is dedicated to assessing the impact of the thermal component of the EOS on the results presented so far, further extending the discussion made in Sec. V. We recall, in fact, that with the exception of the LS220 EOS, all of our EOSs do not have a nuclear-physics thermal component and that thermal effects are accounted for via a hybrid EOS in which an ideal-fluid contribution is added to the total pressure (see Sec. II B for details). The choice of the adiabatic index $\Gamma_{\text {th }}$ is somewhat arbitrary (the only mathematical constraint being that $1 \leq \Gamma_{\text {th }} \leq 2$, but see discussion in [50]). Since the value of $\Gamma_{\text {th }}$ regulates the amount of thermal pressure produced after merger and hence, to some extent, the equilibrium properties of the HMNS, it is worth investigating its effects on the rest-mass density and angularvelocity distributions.

This is shown in Fig. 16 for the ALF2-M125 binary, but the results are similar for other binaries. We indicate with green (black) solid, dashed and dotted lines the averaged angular velocity (rest-mass density) profiles for $\Gamma_{\text {th }}=2.0,1.8$ and 1.6, respectively. The curves are obtained after averaging in the azimuthal direction and over a time interval $\left[1 / 3 t_{\text {fin }}, 2 / 3 t_{\text {fin }}\right]$. Figure 16 shows that a larger value of $\Gamma_{\text {th }}$ yields a larger pres-

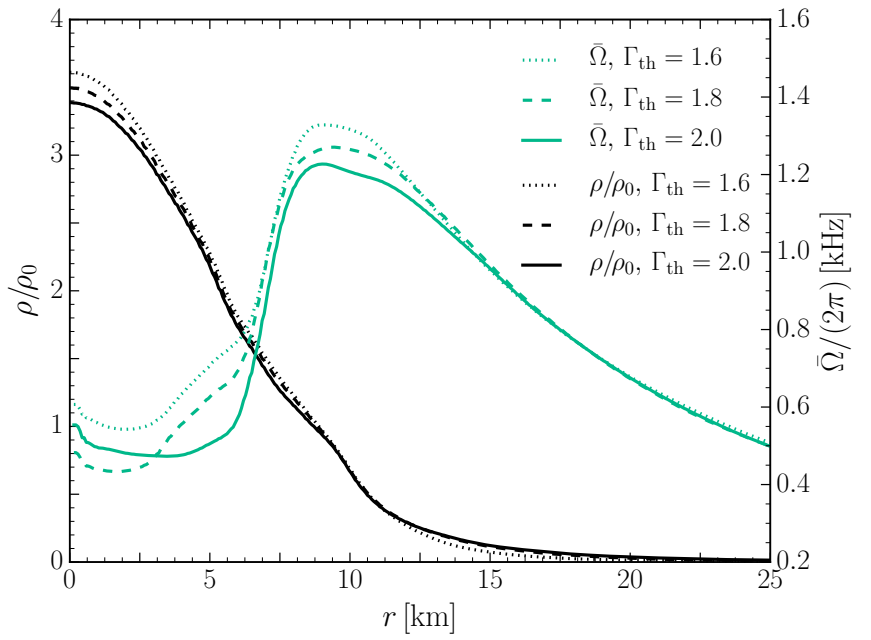

FIG. 16. Time- and azimuthally averaged rest-mass density profiles (black lines) and angular-velocity profiles (green lines) of the ALF2-M125 binary for different values of the thermal adiabatic index $\Gamma_{\text {th }}$. The time and azimuthal averages have been performed in the same manner as in Fig. 12.

sure support and hence prevents the matter in the HMNS to reach large values of compression. This explains why the maximum rest-mass density is larger for smaller values of the thermal adiabatic index (see black lines). In turn, since a larger pressure support implies that the HMNS is less compact (compressed) and since the angular momentum is essentially the same for the binaries with different $\Gamma_{\text {th }}$ considered here, it is not surprising that the maximum value of the averaged angular velocity increases as the contribution of the thermal component is decreased (see green lines).

What is possibly more important to note is that the changes induced by the different values of $\Gamma_{\text {th }}$ are quantitative only and also rather small, i.e., with relative variations of $\lesssim 10 \%$ in the angular velocity. The qualitative behaviour, however, remains unchanged, most notably, in the disk, thus removing the influence of the thermal component of the EOS as a potential bias in our analysis.

\section{CONCLUSIONS}

Establishing the long-term stability properties of astrophysical compact objects produced in catastrophic events, such as in Type-II core collapse supernovae or in the merger of binary systems of neutron stars, is an old and important problem. While it is clear that the large angular momentum that these objects attain cannot be sustained via uniform rotation, far less clear is what is the law of differential rotation that is reached in quasi-stationary equilibria. More importantly, it is not yet known whether this law depends sensitively on the EOS of the compact object or is instead "universal".

Notwithstanding these conceptual obstacles, a large bulk of literature has developed over the last decade to explore this problem in full general relativity, either through the study 


\begin{tabular}{|c|c|c|c|c|c|c|c|}
\hline model & $\begin{array}{c}R_{\Omega_{\max }} \\
{[\mathrm{km}]}\end{array}$ & $\begin{array}{l}\Omega_{\max } \\
{[\mathrm{kHz}]}\end{array}$ & $\begin{array}{c}M_{\mathrm{b}, \Omega_{\max }} \\
{\left[M_{\odot}\right]}\end{array}$ & $\begin{array}{c}M_{\mathrm{b}, \Omega_{\max }} /\left(2 M_{\mathrm{b}, 0}\right) \\
{[\%]}\end{array}$ & $\begin{array}{c}R_{\text {disk }} \\
{[\mathrm{km}]}\end{array}$ & $\begin{array}{c}M_{\mathrm{b}, \text { disk }} \\
{\left[M_{\odot}\right]}\end{array}$ & $\begin{array}{c}M_{\mathrm{b}, \text { disk }} /\left(2 M_{\mathrm{b}, 0}\right) \\
{[\%]}\end{array}$ \\
\hline GNH3-M125 & 7.92 & 1.04 & 1.56 & 57.89 & 18.96 & 0.10 & 3.79 \\
\hline GNH3-M135 & 5.19 & 1.25 & 0.97 & 33.12 & 19.27 & 0.05 & 1.65 \\
\hline H4-M125 & 9.98 & 1.02 & 1.99 & 73.70 & 17.08 & 0.26 & 9.66 \\
\hline $\mathrm{H} 4-\mathrm{M} 135$ & 8.36 & 1.20 & 2.18 & 74.20 & 17.07 & 0.12 & 4.10 \\
\hline ALF2-M125 & 9.04 & 1.23 & 2.04 & 74.49 & 15.71 & 0.32 & 11.73 \\
\hline ALF 2-M135 & 6.20 & 1.31 & 1.46 & 49.35 & 16.39 & 0.04 & 1.35 \\
\hline SLy-M125 & 7.31 & 1.51 & 2.13 & 77.44 & 14.15 & 0.10 & 3.63 \\
\hline SLy-M135 & 6.43 & 1.63 & 2.16 & 72.17 & 15.05 & 0.09 & 3.01 \\
\hline APR4-M125 & 7.57 & 1.52 & 2.15 & 78.49 & 14.56 & 0.12 & 4.43 \\
\hline APR4-M135 & 6.76 & 1.59 & 2.30 & 76.40 & 14.70 & 0.18 & 5.85 \\
\hline
\end{tabular}

TABLE II. Summary of the HMNS properties. The various columns denote the radial position $R_{\Omega_{\max }}$ of the maximum of the averaged angular-velocity profiles $\Omega_{\max }$, the total rest mass inside $R_{\Omega_{\max }}$, i.e., $M_{\mathrm{b}, \Omega_{\max }}:=M_{\mathrm{b}}\left(R_{\Omega_{\max }}\right)$, the radial position $R_{\text {disk }}$ where the disk starts, and the total rest mass outside $R_{\text {disk }}$, i.e., $M_{\mathrm{b}, \text { disk }}:=2 M_{\mathrm{b}, 0}-M_{\mathrm{b}}\left(R_{\mathrm{disk}}\right)$.

of equilibrium configurations or via numerical relativity simulations that produce these objects dynamically. Works in the first class have commonly modelled differential rotation through a particularly simple law expressing that the specific angular momentum is constant on cylinders. In this $j$ - constant law, angular velocity decreases monotonically from the center of the star and the degree of differential rotation is expressed via a single dimensionless parameter. However, a number of simulations of merging binary neutron stars have given evidence that the angular-velocity profile of the HMNS produced at the merger is characterized by a slowly rotating core and an envelope that rotates at frequencies scaling like $r^{-3 / 2}$. This is very different from what is expected when using a $j-$ constant law of differential rotation.

To shed some light on these differences, and to obtain a comprehensive picture of the rotational properties of HMNSs from binary neutron-star mergers, we have carried out a large number of numerical simulations in full general relativity of binary neutron stars described with various EOSs and masses. We have been able to confirm the earlier results of Refs. [4, 19, 20], but, more importantly, to show that the angular-velocity distribution shows only a modest dependence on the EOS, thus exhibiting the traits of "quasi-universality". More specifically, the EOS-independent angular-velocity distributions we find are characterized by an almost uniformly rotating core and a "disk". The rest mass contained in such disk can be quite large, ranging from $\simeq 0.03 M_{\odot}$ in the case of high-mass binaries with stiff EOSs, up to $\simeq 0.2 M_{\odot}$ for low-mass binaries with soft EOSs.

The presence of a disk in the outer regions of the HMNS implies that the disk will only accrete onto the uniformly rotating core on a dissipative timescale, thus not affecting the longterm stability of the latter ${ }^{4}$. The final fate of this disk when the lifetime of the HMNS is expected to be very large (i.e., larger than $10 \mathrm{~s}$ and up to $10^{4} \mathrm{~s} \mathrm{[115])} \mathrm{is} \mathrm{hard} \mathrm{to} \mathrm{assess} \mathrm{through} \mathrm{self-}$

\footnotetext{
${ }^{4}$ By long-term stability we here refer to a timescale which is much longer than the uncertainty in the lifetime of the HMNS due to a finite numerical resolution.
}

consistent numerical simulations. However, its dynamics has been recently conjectured within the "two-winds" model presented in Ref. [34] to explain the extended X-ray emission observed in a class of short gamma-ray bursts. In essence, the expectation is that because the material in the outer regions is on stable orbits, it could be subject to a magnetorotational instability [116-118] and hence behave as a standard accretion disk onto a rapidly rotating magnetized star. In this case, the differential rotation will not brake the rotation but transport angular momentum outwards. A good fraction of the material in this disk will therefore remain on quasi-circular orbits. Once the uniformly rotating core has collapsed, the material in the disk that was not able to accrete before because of the presence of the inner-core "surface", will accrete onto the black hole on the timescale set by the most efficient dissipative mechanism removing angular momentum. Clearly, if the mass in the torus is very small, then it will become difficult to find the energy reservoir needed to launch and sustain the jet that is required in the two-winds model. However, given that at least $10 \%$ of a solar mass is present in the disk after $20 \mathrm{~ms}$ after the merger, it is sufficient that only $10 \%$ of this mass is channelled into a torus around the newly formed black hole to provide a sufficient amount of energy to power a relativistic jet. More work is needed to fully explore the consequences of this scenario and also to assess the impact that neutrino-driven winds may have on the survival of the disk [119].

On a final note, we should also draw attention to various limitations of the work presented here. First, all of the binaries considered here have the same mass; although the masses in observed neutron-star binaries do not differ significantly, it is unlikely that they are exactly the same. Fortunately, it seems that this systematic bias in our sample may not be a serious one since the results presented in Ref. [120] for unequal-mass binaries show very similar angular-velocity profiles. Second, our simulations do not account for neutrino transfer; this is mostly because the neutrino-diffusion timescale is at least one order of magnitude larger than the one considered here [121] and a complete investigation of the rotational properties of the HMNS when varying the EOS and the neutrino transport are still prohibitive. Hence, we have preferred to consider here a 
more controlled scenario in which we evaluate only the impact of the EOS. Third, our simulations have neglected magnetic fields, even though we do expect magnetic fields to impact the dynamics of the HMNS by transferring angular momentum from the inner regions of the HMNS out to the more external ones. This transfer will take place on an Alfvén timescale, which is much longer than the one considered here. In this sense, the quasi-universal behaviour reported here should be taken as representative of the first few tens of milliseconds after the merger and recent work in Ref. [120] indicates that our results should remain valid also in the presence of magnetic fields and at least within $\sim 40 \mathrm{~ms}$ after merger. The importance of magnetic fields in the post-merger dynamics has been discussed in recent works [17, 122-127]. During merger a vortex sheet develops where the tangential component of the velocity is discontinuous. Such a shear interface is unstable to perturbations and can develop the so-called Kelvin-Helmholtz instability. If the magnetic-field of the merging neutron stars is poloidal, this instability may lead to an exponential growth of their toroidal component [13, 17, 122, 123, 128, 129], curling poloidal field lines and generating a turbulent flow. Resolving the growth and saturation of the Kelvin-Helmholtz instability is a challenging computational issue, especially for realistic (pulsar-like) magnetic field strengths. The latest, highest resolution simulations to date of [123] for initial magnetic fields of moderate strength $\left(10^{13} \mathrm{G}\right)$ show that the amplification factor of the field is $\sim 10^{3}$ at $\sim 4 \mathrm{~ms}$ after merger, with a saturation magnetic field-energy $\gtrsim 4 \times 10^{50} \mathrm{erg}$, i.e., $\gtrsim 10^{-3}$ of the bulk kinetic energy of the merging neutron stars. Although the magnetic energy is still much smaller than the bulk kinetic energy and unable to produce significant changes in the inspiral [130], these results and the possibility that even stronger amplification could be achieved, suggest the importance to consider strong magnetic fields for modelling the post-merger evolution of binary neutron stars. Finally, our investigation has been concentrated mostly on "cold" EOSs, where a thermal contribution has been added in a way that is "ad-hoc", albeit quite customary [cf. Eq. (6)]. It is therefore reassuring that two of the 12 binaries simulated here, and for which a "hot" EOS has been employed, show a behaviour for the angular velocity that is very similar to that encountered for the cold EOSs. Overall, the caveats listed above represent strong motivations to further refine the study carried out here and advance our understanding of the rotational properties of the HMNSs produced in binary neutron-star mergers.

\section{ACKNOWLEDGEMENTS}

We thank B. Mundim, N. Gürlebeck, M. G. Alford, W. Kastaun for useful discussions and comments. Support comes from the ERC Synergy Grant "BlackHoleCam" (Grant 610058), from "NewCompStar", COST Action MP1304, from the LOEWE-Program in HIC for FAIR, from the European Union's Horizon 2020 Research and Innovation Programme (Grant 671698) (call FETHPC-1-2014, project ExaHyPE), from JSPS KAKENHI grant (Grant 15H06813), from the Spanish MINECO (grants AYA2013-40979-P and
AYA2015-66899-C2-1-P), and from the Generalitat Valenciana (PROMETEOII-2014-069). MH gratefully acknowledges support from the Frankfurt Institute for Advanced Studies (FIAS) and the Goethe University Frankfurt, while HS acknowledges the Judah M. Eisenberg laureatus Professur endowment. The simulations were performed on SuperMUC at LRZ-Munich, on LOEWE at CSC-Frankfurt and on Hazelhen at HLRS in Stuttgart.

\section{Appendix A: On the Bernoulli constant}

As discussed in section III B, the evident $\pi / 2$ phase difference in the distribution of the angular velocity and of the density, so that areas of low pressure (rest-mass density) are accompanied by regions of large velocity, can be explained in terms of the manifestation of the Bernoulli theorem. To show this, we will consider a rather idealised description of the quasi-stationary equilibrium of the HMNS and make a series of assumptions that will simplify the mathematical treatment and hopefully improve our physical understanding.

We therefore start by recalling that in relativistic hydrodynamics and for a perfect fluid with four-velocity $\boldsymbol{u}$, the quantity $h(\boldsymbol{u} \cdot \boldsymbol{\xi})$ is Lie-dragged along $\boldsymbol{u}$ [50]

$$
\mathscr{L}_{\boldsymbol{u}}(h \boldsymbol{u} \cdot \boldsymbol{\xi})=0
$$

where $h:=(e+p) / \rho$ is the specific enthalpy, $e:=\rho(1+\epsilon)$ is the total energy density, and $\boldsymbol{\xi}$ is a Killing vector of the spacetime and also a generator of the symmetry obeyed by the fluid. A direct consequence of Eq. (A1) is that in the case in which the spacetime admits a timelike Killing vector, then the quantity $\mathcal{B}:=h u_{t}$ is a constant of the fluid; this is the generalrelativistic extension of the classical Bernoulli theorem.

Of course, the assumption of a stationary spacetime during the evolution of the HMNS is not true and the HMNS is emitting gravitational waves, which are the most evident manifestation of a non-stationary spacetime. Yet, because at least energetically these modulations of the spacetime are small when compared with the total bulk (kinetic) energy of the system, we can consider the assumption not to be unreasonable even though it is evidently not strictly true.

In its classical limit, Eq. (A1) becomes

$$
\left(1+\epsilon+\frac{p}{\rho}\right)\left(1+\phi+\frac{1}{2} \overrightarrow{\boldsymbol{v}}^{2}\right)=\text { const. },
$$

where $\phi$ is the gravitational potential and where $\boldsymbol{v}$ is the local fluid velocity. When neglecting higher-order terms, expression (A2) further reduces to

$$
\left(\frac{1}{2} \overrightarrow{\boldsymbol{v}}^{2}+\phi+\epsilon+\frac{p}{\rho}\right)=\text { const. }
$$

which coincides with the classical expression for the Bernoulli constant [50]. Next, to translate into a classical Newtonian language our assumption on the existence of a timelike Killing vector we can take the gravitational potential to be independent of time and essentially constant across the HMNS, so 


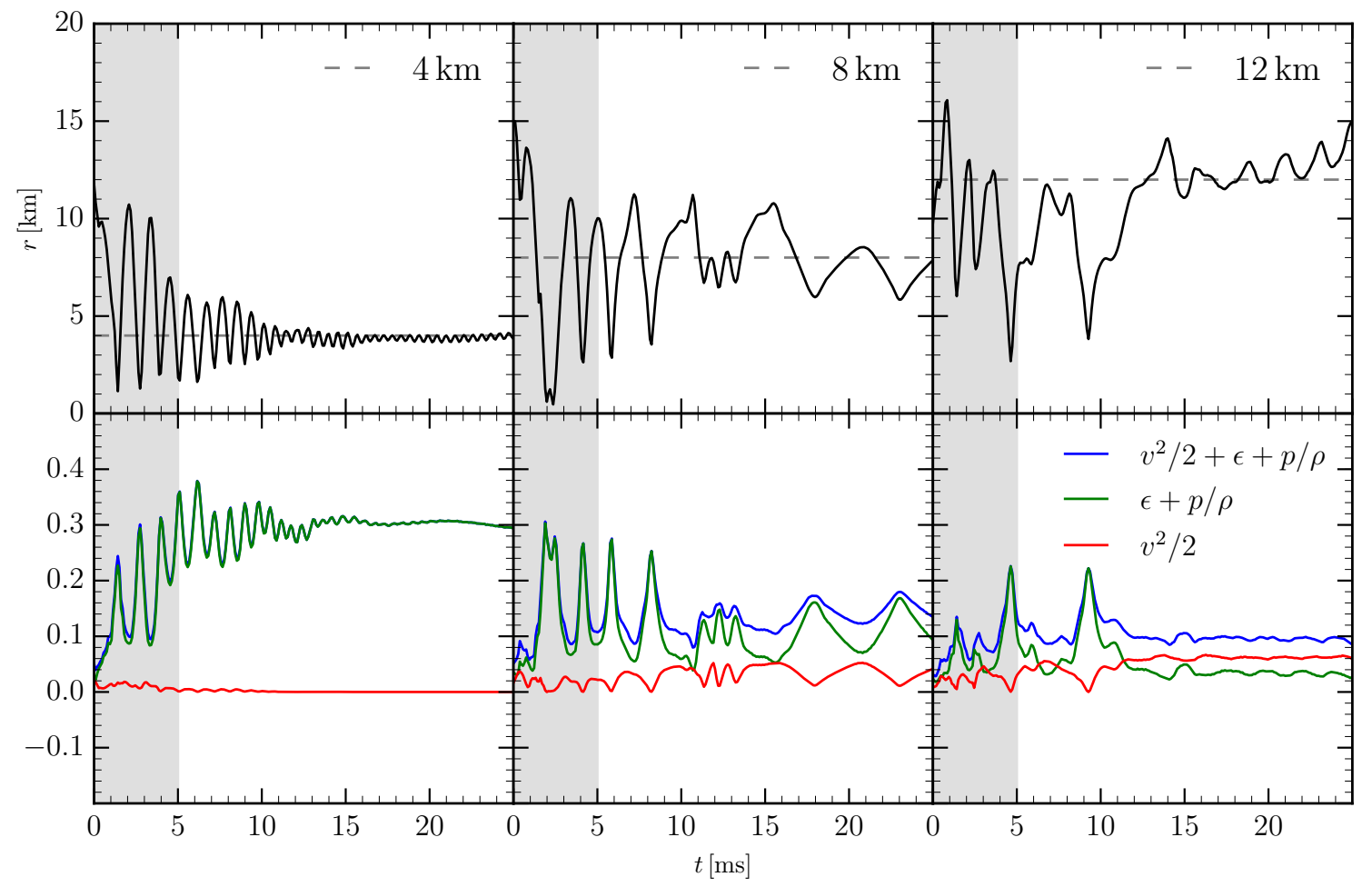

FIG. 17. Top panels: evolution of the radial positions for tracers in the LS220-M132 binary that are eventually either in the inner regions of the HMNS (left panel), or at some distance from the rotation axis (central panel), or in outer regions of the HMNS (right panel). Bottom panels: evolution of the classical Bernoulli constant (A4) relative to these tracers (blue solid lines), and its main contributions. The gray-shaded area refers to the post-merger transient when the HMNS is far from an equilibrium.

that Bernoulli's theorem effectively reduces to the well-known condition that, along a fluidline,

$$
\left(\frac{1}{2} \overrightarrow{\boldsymbol{v}}^{2}+\epsilon+\frac{p}{\rho}\right)=\text { const. . }
$$

To validate whether or not the classical Bernoulli constant (A4) is actually a constant along a fluidline we have calculated it for a number of tracer particles and show it for three representative fluidlines in Fig. 17 for the LS220-M132 binary. These tracers have been selected because they are originally in the equatorial plane and have an essentially zero velocity in the vertical rotation, hence representing particles that are genuinely moving in the equatorial plane. The three panels in the top row of Fig. 17 show the evolution of the radial positions for tracers that at the end of the simulation are either in the inner regions of the HMNS [i.e., $r\left(t=t_{\text {fin }}\right)=4 \mathrm{~km}$, left panel], or at some distance from the rotation axis [i.e., $r(t=$ $\left.t_{\text {fin }}\right)=8 \mathrm{~km}$, central panel], or in outer regions of the HMNS [i.e., $r\left(t=t_{\text {fin }}\right)=12 \mathrm{~km}$, right panel]. The gray-shaded area refers to the post-merger transient when the HMNS is far from an equilibrium. Note that these selected particles can experience large excursions from their original positions (shown as dashed horizontal lines) due to the complex motion around the core per Fig. 10, but that on average they do not stride too far away.
The panels in the bottom row of Fig. 17 show instead the values of the classical Bernoulli constant (A4) relative to the corresponding tracers in the top row (blue solid lines), but also the two main quantities contributing to it, namely: $\epsilon+p / \rho$ (green solid lines) $^{5}$ and $v^{2} / 2$ (red solid lines). While the values of (A4) are strictly not constant in time (especially in transient post-merger phase indicated with the gray-shaded areas), they also do not vary significantly around the initial values. More importantly, it is very clear that there is a phase opposition in the evolution of the pressure term $\epsilon+p / \rho$ and of the kinetic term $v^{2} / 2$, so that large values of the former correspond to low values of the latter and viceversa. This is exactly what one would expect in the presence of a fluid satisfying Bernoulli's theorem, hence supporting the explanation of the phase difference in the distribution of angular velocity, recalling that $v \sim \Omega r$, and density being a result of the conservation of the Bernoulli quantity $\mathcal{B}=h u_{t}$. All that has been discussed above for our three representative tracers holds true for all others that are taken in the neighborhood of the equatorial plane.

\footnotetext{
${ }^{5}$ Note that $\epsilon \simeq p / \rho$ at all times and hence they are not shown separately.
} 

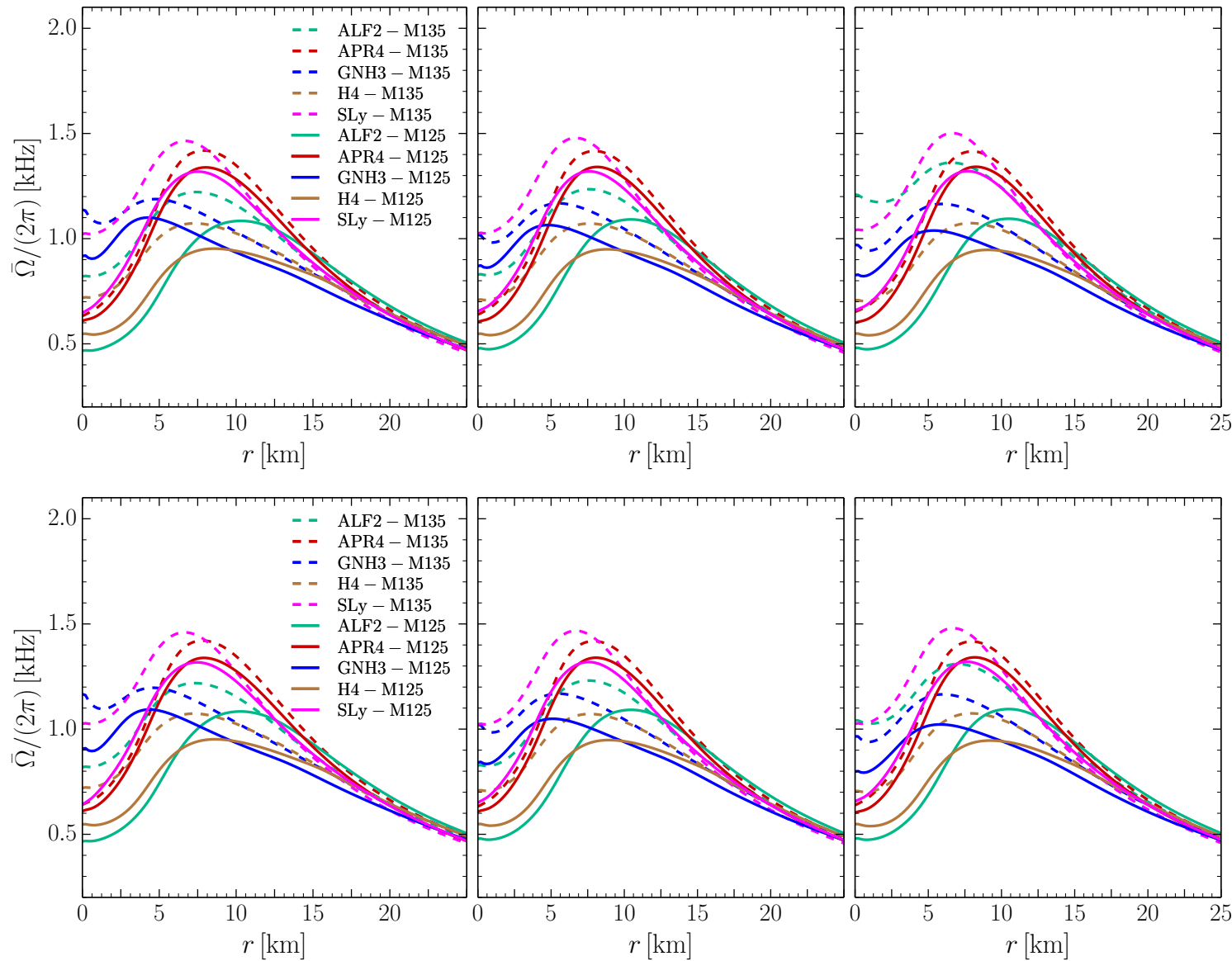

FIG. 18. Comparison of different averaging techniques for the time- and azimuthally averaged angular-velocity profiles for different EOSs. The beginning of the averaging window has been fixed at $6 \mathrm{~ms}$. Top panels: For all EOSs and masses, the different panels refer to different lengths of the window, namely, $5,7,9 \mathrm{~ms}$, so that the from left to right the data refer to time windows $[6,11],[6,13]$ and $[6,15]$ ms, respectively. Bottom panels: The same as above but when the averaging window is not the same for the different EOSs and masses but is determined by the gravitational-wave frequency $f_{2}=\Omega_{2} /(2 \pi)$. As a result, from left to right the averaging windows are: $[12,18,24] \times 1 / f_{2}$, respectively.

\section{Appendix B: Time-averaging, symmetries, and resolutions}

In this Appendix we consider the impact that the timeaveraging techniques, the use of a $\pi$-symmetry and the chosen spatial resolution have on the robustness of our results. We recall that all the simulations reported here have used six refinement levels and a rather high spatial resolution, namely, $\Delta h_{5}=0.15 M_{\odot} \approx 221 \mathrm{~km}$ on the finest refinement level. Furthermore, to reduce computational costs, we have employed a reflection symmetry across the $z=0$ plane and for most simulations a $\pi$-symmetry condition across the $x=0$ plane.

\section{Impact of time-averaging techniques}

The choice of the origin and length of the time-averaging window has been guided by two principal considerations: avoiding the initial post-merger phase and avoiding the phase briefly preceding the collapse to a black hole. Avoiding the initial post-merger phase is important because the HMNS is rapidly changing in its attempt to reach an equilibrium; the matter dynamics in this phase is quite irregular, as can be seen in the gravitational waves [30], and differs significantly from the evolution at later times when the system reaches a more equilibrium state. On the other hand, avoiding the stage preceding the collapse to a black hole is important because again in such a stage the dynamics is far from equilibrium and any information on the angular velocity does not reflect a quasistationary solution.

Within these constraints, there are two free parameters in performing the time averages: the initial time of the averaging window and its width. We recall that in the previous section we have chosen a time-averaging centered about $t_{\text {fin }} / 2$ with a width of $t_{\text {fin }} / 3$ where $t_{\text {fin }}$ is the time to collapse to a black hole in the high-mass cases and the end of the simulation in the low-mass cases. These values ensure that the above mentioned considerations are realised and have no influence on the angular-velocity profiles. Although these constraints may appear restrictive, we consider next different times and how 


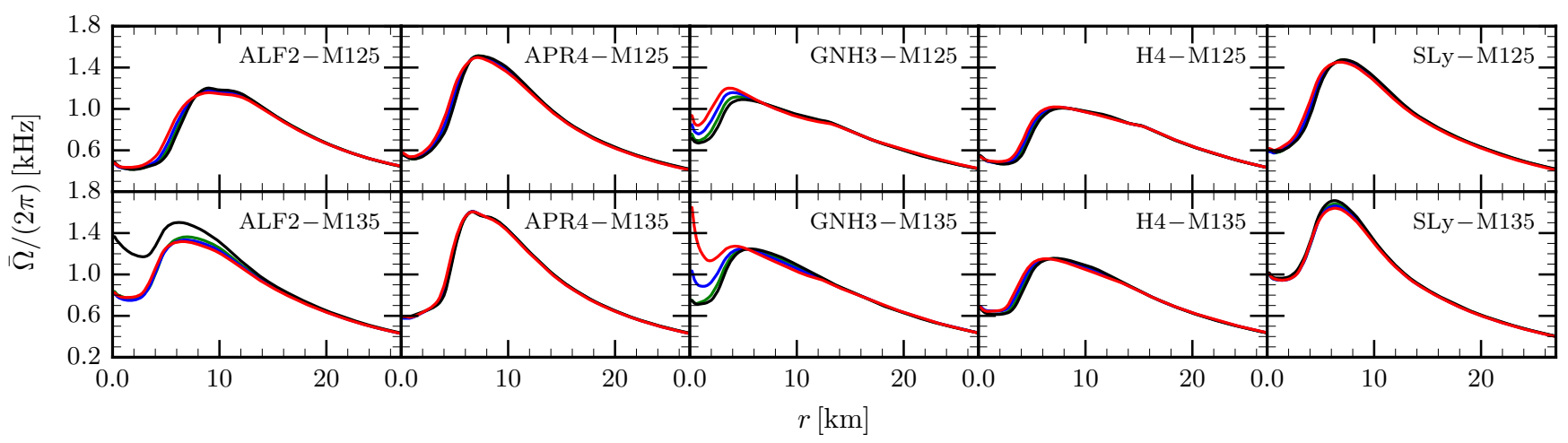

FIG. 19. Averaged angular-velocity profiles when the avering windows is set to be $7 \mathrm{~ms}$ for all EOSs and masses, but where the initial averaging time is varied and set to be 5 (red line), 6 (blue line), 7 (green line), and $8 \mathrm{~ms}$ (black line), respectively. The four lines refer to averaging windows given by $[5,12],[6,13],[7,14]$, and $[8,15] \mathrm{ms}$, respectively; note that the top part of each panel refers to the low-mass binary, while the bottom one to the high-mass one.

they influence the averaging procedure.

To this end, we have chosen several initial and final values of the averaging procedure and plotted the results in Fig. 18. More precisely, the top panels of Fig. 18 report the angularvelocity profiles when the initial time of the averaging window is taken to be $6 \mathrm{~ms}$ after the merger, independently of the EOS and mass of the binary. Furthermore, the different panels from left to right refer to different lengths of the window, namely, $5,7,9 \mathrm{~ms}$, so that the windows data refer to time windows $[6,11],[6,13]$ and $[6,15] \mathrm{ms}$, respectively. Clearly, independent of the window length, the qualitative features are essentially identical, exhibiting a slowly rotating core, followed by an increase to a maximum, followed by a decrease to a flow with $\Omega(r) \propto r^{-3 / 2}$.

The bottom panels of Fig. 18, on the other hand, show a similar information in that the initial time is still fixed to $6 \mathrm{~ms}$, but the averaging window is not the same for the different EOSs and masses. Rather, it is determined by the gravitational-wave frequency $f_{2}=\Omega_{2} /(2 \pi)$, which is related to the maximum of the angular velocity $\Omega_{\max }$ (cf. Fig. 13). In this way, each binary will have an average window which is set to be a multiple of the spinning frequency of the HMNS. In practice we have set the averaging window to be $\Delta t=[12,18,24] \times 1 / f_{2}$ in the panels from left to right, respectively. Also in this case, the qualitative behaviour of the various angular-velocity profiles is the same and the differences are of a few percent at most.

As a final variant of the possible way of performing the time averages, we report in Fig. 19 the angular-velocity profiles when the averaging windows is set to be $7 \mathrm{~ms}$ for all EOSs and masses, but where the initial averaging time is varied and set to be 5, 6, 7 and $8 \mathrm{~ms}$, respectively. As a result, the four lines reported in each panel refer to averaging windows given by $[5,12],[6,13],[7,14]$, and $[8,15] \mathrm{ms}$, respectively; note that the top part of each panel refers to the low-mass binary, while the bottom one to the high-mass binary. Also when considering this different technique it emerges rather clearly that the averaging procedure has little influence on the angular-velocity distribution. However, two exceptions are also equally clear and for obvious reasons. The first one is offered by the binary ALF2-M135 case, whose HMNS collapses at approximately $15 \mathrm{~ms}$ (cf. Fig. 2) and whose "latetime" averaging window is obviously spoiled by the large increase in $\Omega$ occurring before the collapse. The second exception is given instead by the binary GNH3-M1 35, which has instead a long-lasting transient post-merger phase, with the two stellar cores still clearly visible. Also in this, the "early-time" averaging is not representative of a quasi-stationary stage. Excluding these two obvious pathological averaging windows, the maximum angular-velocity changes by $5 \%$ at most for all masses and EOSs.

\section{Impact of $\pi$-symmetry}

We next consider the impact of having imposed a $\pi$ symmetry in our simulations. While this is a perfectly reasonable option in view of the considerable savings in computational costs, it also blinds us to the development of an $m=1$ instability that has been reported by a number of groups [131133]. While the gravitational-wave signal associated with the instability is always smaller than the dominant one coming from the $m=2$ deformations in the HMNS, so that its observation by current generation detectors is unlikely and will require third-generation detectors [132], it is useful to verify whether the presence of the one-arm instability would leave an imprint on the angular-velocity profiles despite the azimuthal average.

To this scope we have considered the evolution of an equalmass binary with the LS220 EOS and a gravitational mass of $2 \times 1.350 M_{\odot}$ (cf. binary LS220-M135 in Table I), evolved with and without $\pi$-symmetry to investigate the influence of the instability on the rotation profiles. The corresponding angular-velocity distribution on the equatorial plane for the simulation without the $\pi$-symmetry is shown in the left panel of Fig. 20 at four representative times after the merger and 

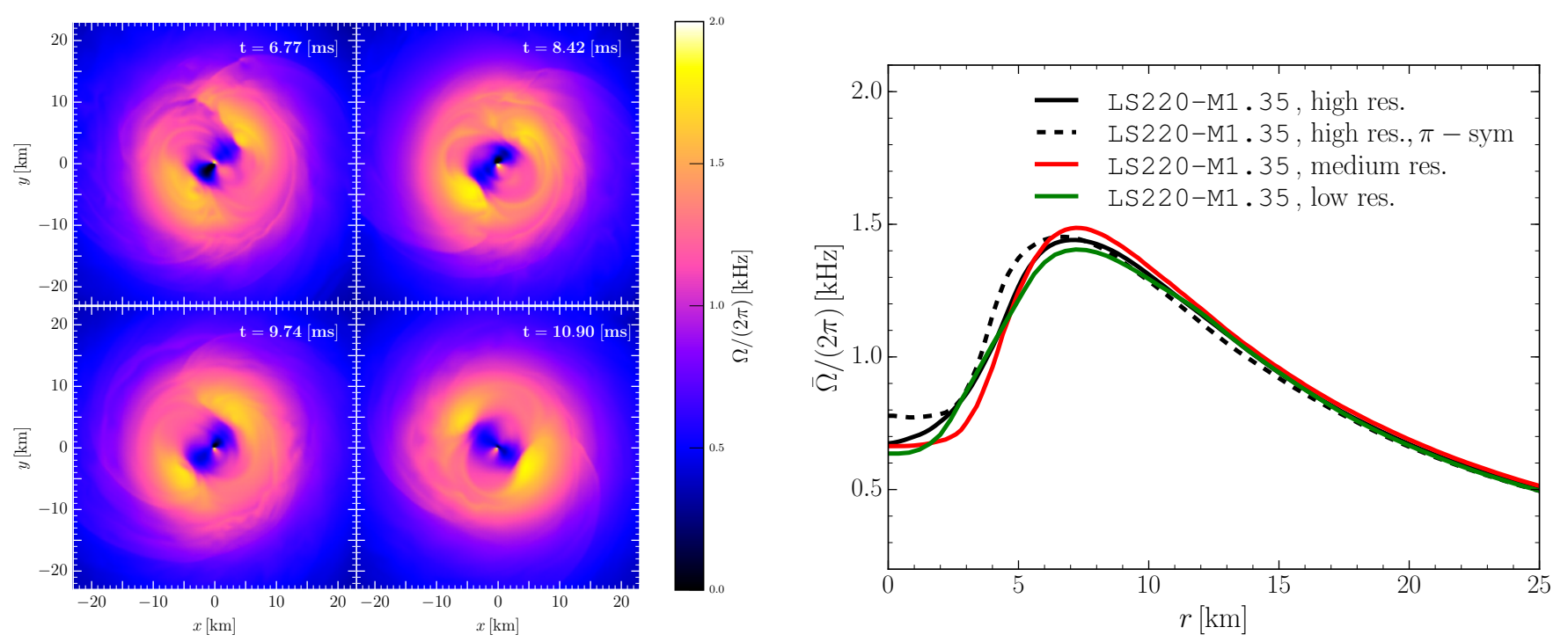

FIG. 20. Left panel: angular velocity distribution on the equatorial plane at four representative times for a binary with the LS220 EOS evolved without $\pi$-symmetry; note the appearance of an $m=1$ deformation in addition to the larger $m=2$ deformation. Right panel: corresponding azimuthal and time-averaged profile for the same binary with $\pi$-symmetry (black dashed line) and without (black solid line) at a resolution of $\Delta x=0.15$. Additionally, low resolution runs of $\Delta x=0.20$ (red solid line) and $\Delta x=0.25$ (green solid line) are shown. All resolutions exhibit the same behaviour already discussed above.

when the HMNS has reached a quasi-stationary state. Comparing such a panel with the bottom rows of Figs. 3 and 9, where the $\pi$-symmetry is imposed, highlights the presence of a small $m=1$ deformation. The right panel of Fig. 20, on the other hand, reports the corresponding azimuthal and timeaveraged profile for two simulations. The black dashed line refers to the $\pi$-symmetric run, while the black solid line to the run without $\pi$-symmetry; in both cases the average is done between $t=5 \mathrm{~ms}$ and $t=12 \mathrm{~ms}$.

Clearly, no sign of the $m=1$ deformation is present, as one would expect from an averaging process; rather, the angular velocity shows similar quantitative behaviour with and without the use of $\pi$-symmetry. The greatest difference is in the very centre of the HMNS where the angular velocity is higher with $\pi$-symmetry than without. The maximum angular velocity is $1 \%$ larger with $\pi$-symmetry and the location of the maximum is slightly shifted to larger radii. Both runs exhibit quasi-circular orbits at larger radii. We conclude that in the very interior of the HMNS, the use of $\pi$-symmetry plays a small role, but also that outside a core region of $\simeq 5 \mathrm{~km}$ the influence is minimal and does not affect our conclusions.

\section{Impact of grid resolution}

Since the stability properties of the HMNS phase depend on the resolution (see [37] where this was first investigated systematically), the determination of its lifetime against gravitational collapse requires a systematic and very careful resolution study. At the same time, because of the development of large shocks, the convergence order after the merger is inevitably very low (i.e., of order unity or less), so that a strict convergence study of this stage is of little use and certainly beyond the scope of this paper. In fact, different resolutions would mostly produce phase differences in the dynamics of the fluid and spacetime variables, hence with only a small impact on our results that are expressed in terms of time and azimuthal averages.

Notwithstanding these considerations, it is reasonable to ask how significant are the changes in the angular-velocity profiles when the simulations are performed at different resolutions. Such a resolution study would then provide confidence on the robustness of the results presented here. We also recall that in Ref. [36] we have considered the influence of the resolution on the dynamics of the HMNS by using three different resolutions for a binary described by an ideal-fluid EOS. As remarked in [36], the rather high resolution employed here on the finest refinement level, i.e., $\Delta h_{5}=0.15 M_{\odot}$, provides a description of the HMNS which is very close to that obtained with an even higher resolution of $\Delta h_{5}=0.125 M_{\odot}$.

The results of our resolution study are summarized in the right panel of Fig. 20 for the LS220-M135 binary. More specifically, in the right panel of Fig. 20 we plot the averaged angular velocity for the binary LS220-M135 for three different resolutions on the finest refinement level, i.e., $\Delta h_{5}=$ $0.15,0.20,0.25 M_{\odot} \approx 221,295,369 \mathrm{~m}$, and which we dub as "high", "medium" and "low resolution", respectively. All simulations do not use a $\pi$-symmetry, except where noted. We also remark that although a resolution of $\Delta x=0.25 M_{\odot}$ may appear coarse, it is routinely used in numerical-relativity simulations of binary neutron stars (see, e.g., [18, 134, 135]) and has been shown to be high enough to provide physically robust results (see [18] for an extensive discussion).

Clearly, at all resolutions the profile of the angular-velocity 
is similar, namely, showing a slowly rotating core, rising to a maximum around $8 \mathrm{~km}$ before decreasing to a $r^{-3 / 2}$ profile. The largest differences between resolution are in the centre of the HMNS, where the rest-mass densities are the highest and the metric functions show the largest gradients. Despite this, all resolutions reach a maximum angular-velocity at around $8 \mathrm{~km}$ with a variation with resolution that is at most $5 \%$. This small variance demonstrates the robustness of the maximum angular-velocity and illustrates that quasi-universal relations proposed in Sec. VI are a robust feature of the HMNS.
[1] T. W. Baumgarte, S. L. Shapiro, and M. Shibata, Astrophys. J. Lett. 528, L29 (2000), arXiv:astro-ph/9910565

[2] N. D. Lyford, T. W. Baumgarte, and S. L. Shapiro, Astrophys. J. 583, 410 (2003), gr-qc/0210012

[3] M. Shibata, K. Taniguchi, and K. Uryū, Phys. Rev. D 71, 084021 (2005), gr-qc/0503119

[4] M. Shibata and K. Taniguchi, Phys. Rev. D 73, 064027 (2006), astro-ph/0603145

[5] M. Ansorg, D. Gondek-Rosińska, and L. Villain, Mon. Not. R. Astron. Soc. 396, 2359 (2009), arXiv:0812.3347 [gr-qc]

[6] F. Galeazzi, S. Yoshida, and Y. Eriguchi, Astron. Astrophys. 541, A156 (2012), arXiv:1101.2664 [astro-ph.SR]

[7] A. M. Studzińska, M. Kucaba, D. Gondek-Rosińska, L. Villain, and M. Ansorg, Mon. Not. R. Astron. Soc. 463, 2667 (2016)

[8] D. Gondek-Rosinska, I. Kowalska, L. Villain, M. Ansorg, and M. Kucaba, arXiv:1609.02336 (2016), arXiv:1609.02336 [astro-ph.HE]

[9] C. D. Ott, A. Burrows, T. A. Thompson, E. Livne, and R. Walder, Astrophys. J. Suppl. Ser. 164, 130 (2006), astro$\mathrm{ph} / 0508462$

[10] L. Baiotti and L. Rezzolla, arxiv:1607.03540 (2016), arXiv: 1607.03540 [gr-qc]

[11] C. Breu and L. Rezzolla, Mon. Not. R. Astron. Soc. 459, 646 (2016), arXiv:1601.06083 [gr-qc]

[12] M. Shibata and K. Uryū, Phys. Rev. D 61, 064001 (2000), grqc/9911058

[13] L. Baiotti, B. Giacomazzo, and L. Rezzolla, Phys. Rev. D 78, 084033 (2008), arXiv:0804.0594 [gr-qc]

[14] M. Anderson, E. W. Hirschmann, L. Lehner, S. L. Liebling, P. M. Motl, D. Neilsen, C. Palenzuela, and J. E. Tohline, Phys. Rev. D 77, 024006 (2008), arXiv:0708.2720 [gr-qc]

[15] Y. T. Liu, S. L. Shapiro, Z. B. Etienne, and K. Taniguchi, Phys. Rev. D 78, 024012 (2008), arXiv:0803.4193 [astro-ph]

[16] S. Bernuzzi, M. Thierfelder, and B. Brügmann, Phys. Rev. D 85, 104030 (2012), arXiv:1109.3611 [gr-qc]

[17] L. Rezzolla, B. Giacomazzo, L. Baiotti, J. Granot, C. Kouveliotou, and M. A. Aloy, Astrophys. J. Letters 732, L6 (2011), arXiv:1101.4298 [astro-ph.HE]

[18] R. De Pietri, A. Feo, F. Maione, and F. Löffler, Phys. Rev. D 93, 064047 (2016), arXiv:1509.08804 [gr-qc]

[19] W. Kastaun and F. Galeazzi, Phys. Rev. D 91, 064027 (2015), arXiv:1411.7975 [gr-qc]

[20] W. Kastaun, R. Ciolfi, and B. Giacomazzo, Phys. Rev. D 94 , 044060 (2016), arXiv:1607.02186 [astro-ph.HE]

[21] W. Kastaun, F. Galeazzi, D. Alic, L. Rezzolla, and J. A. Font, Phys. Rev. D 88, 021501 (2013), arXiv:1301.7348 [gr-qc]

[22] K. Yagi and N. Yunes, Phys. Rev. D 88, 023009 (2013), arXiv:1303.1528 [gr-qc]

[23] G. Pappas and T. A. Apostolatos, Phys. Rev. Lett. 112, 121101 (2014), arXiv:1311.5508 [gr-qc]

[24] L. C. Stein, K. Yagi, and N. Yunes, Astrophys. J. 788, 15 (2014), arXiv:1312.4532 [gr-qc]

[25] B. Haskell, R. Ciolfi, F. Pannarale, and L. Rezzolla, Mon. Not.
R. Astron. Soc. Letters 438, L71 (2014), arXiv:1309.3885 [astro-ph.SR]

[26] D. D. Doneva, S. S. Yazadjiev, N. Stergioulas, and K. D. Kokkotas, Astrophys. J. Letters 781, L6 (2014), arXiv:1310.7436 [gr-qc]

[27] S. Chakrabarti, T. Delsate, N. Gürlebeck, and J. Steinhoff, Phys. Rev. Lett. 112, 201102 (2014), arXiv:1311.6509 [gr-qc]

[28] A. Maselli, V. Cardoso, V. Ferrari, L. Gualtieri, and P. Pani, Phys. Rev. D 88, 023007 (2013), arXiv:1304.2052 [gr-qc]

[29] K. Takami, L. Rezzolla, and L. Baiotti, Phys. Rev. Lett. 113, 091104 (2014), arXiv:1403.5672 [gr-qc]

[30] L. Rezzolla and K. Takami, Phys. Rev. D 93, 124051 (2016), arXiv: 1604.00246 [gr-qc]

[31] B. Zhang and P. Mészáros, Astrophys. J. 552, L35 (2001), astro-ph/0011133

[32] B. D. Metzger, E. Quataert, and T. A. Thompson, Mon. Not. R. Astron. Soc. 385, 1455 (2008), arXiv:0712.1233

[33] N. Bucciantini, B. D. Metzger, T. A. Thompson, and E. Quataert, Mon. Not. R. Astron. Soc. 419, 1537 (2012), arXiv:1106.4668 [astro-ph.HE]

[34] L. Rezzolla and P. Kumar, Astrophys. J. 802, 95 (2015), arXiv: 1410.8560 [astro-ph.HE]

[35] R. Ciolfi and D. M. Siegel, Astrophys. J. 798, L36 (2015), arXiv:1411.2015 [astro-ph.HE]

[36] K. Takami, L. Rezzolla, and L. Baiotti, Phys. Rev. D 91, 064001 (2015), arXiv:1412.3240 [gr-qc]

[37] L. Baiotti, B. Giacomazzo, and L. Rezzolla, Class. Quantum Grav. 26, 114005 (2009), arXiv:0901.4955 [gr-qc]

[38] L. Baiotti, M. Shibata, and T. Yamamoto, Phys. Rev. D 82, 064015 (2010), arXiv: 1007.1754 [gr-qc]

[39] D. Brown, P. Diener, O. Sarbach, E. Schnetter, and M. Tiglio, Phys. Rev. D 79, 044023 (2009), arXiv:0809.3533 [gr-qc]

[40] F. Löffler, J. Faber, E. Bentivegna, T. Bode, P. Diener, R. Haas, I. Hinder, B. C. Mundim, C. D. Ott, E. Schnetter, G. Allen, M. Campanelli, and P. Laguna, Class. Quantum Grav. 29, 115001 (2012), arXiv:1111.3344 [gr-qc]

[41] The Einstein Toolkit Consortium: einsteintoolkit. org

[42] T. Nakamura, K. Oohara, and Y. Kojima, Progress of Theoretical Physics Supplement 90, 1 (1987)

[43] M. Shibata and T. Nakamura, Phys. Rev. D 52, 5428 (1995)

[44] T. W. Baumgarte and S. L. Shapiro, Phys. Rev. D 59, 024007 (1999), gr-qc/9810065

[45] M. Alcubierre, B. Brügmann, P. Diener, M. Koppitz, D. Pollney, E. Seidel, and R. Takahashi, Phys. Rev. D 67, 084023 (2003), gr-qc/0206072

[46] D. Pollney, C. Reisswig, L. Rezzolla, B. Szilágyi, M. Ansorg, B. Deris, P. Diener, E. N. Dorband, M. Koppitz, A. Nagar, and E. Schnetter, Phys. Rev. D 76, 124002 (2007), arXiv:0707.2559 [gr-qc]

[47] L. Baiotti, I. Hawke, P. J. Montero, F. Löffler, L. Rezzolla, N. Stergioulas, J. A. Font, and E. Seidel, Phys. Rev. D 71, 024035 (2005), gr-qc/0403029

[48] L. Rezzolla, L. Baiotti, B. Giacomazzo, D. Link, and 
J. A. Font, Class. Quantum Grav. 27, 114105 (2010), arXiv:1001.3074 [gr-qc]

[49] L. Baiotti, T. Damour, B. Giacomazzo, A. Nagar, and L. Rezzolla, Phys. Rev. Lett. 105, 261101 (2010), arXiv:1009.0521 [gr-qc]

[50] L. Rezzolla and O. Zanotti, Relativistic Hydrodynamics (Oxford University Press, Oxford, UK, 2013)

[51] J. A. Font, Living Rev. Relativ. 6, 4; http://www.livingreviews.org/lrr (2008), 0704.2608.

[52] A. Harten, P. D. Lax, and B. van Leer, SIAM Rev. 25, 35 (1983)

[53] P. Colella and P. R. Woodward, Journal of Computational Physics 54, 174 (1984)

[54] E. Schnetter, S. H. Hawley, and I. Hawke, Class. Quantum Grav. 21, 1465 (2004), gr-qc/0310042

[55] E. Gourgoulhon, P. Grandclément, K. Taniguchi, J.-A. Marck, and S. Bonazzola, Phys. Rev. D 63, 064029 (2001), grqc/0007028

[56] H.-T. Janka, T. Zwerger, and R. Mönchmeyer, Astron. Astrophys. 268, 360 (1993)

[57] A. Akmal, V. R. Pandharipande, and D. G. Ravenhall, Phys. Rev. C 58, 1804 (1998), arXiv:hep-ph/9804388

[58] F. Douchin and P. Haensel, Astron. Astrophys. 380, 151 (2001), arXiv:astro-ph/0111092

[59] N. K. Glendenning, Astrophys. J. 293, 470 (1985)

[60] N. K. Glendenning and S. A. Moszkowski, Phys. Rev. Lett. 67, 2414 (1991)

[61] M. Alford, M. Braby, M. Paris, and S. Reddy, Astrophys. J. 629, 969 (2005), nucl-th/0411016

[62] J. Antoniadis, P. C. C. Freire, N. Wex, T. M. Tauris, R. S. Lynch, M. H. van Kerkwijk, M. Kramer, C. Bassa, V. S. Dhillon, T. Driebe, J. W. T. Hessels, V. M. Kaspi, V. I. Kondratiev, N. Langer, T. R. Marsh, M. A. McLaughlin, T. T. Pennucci, S. M. Ransom, I. H. Stairs, J. van Leeuwen, J. P. W. Verbiest, and D. G. Whelan, Science 340, 448 (2013), arXiv:1304.6875 [astro-ph.HE]

[63] J. S. Read, B. D. Lackey, B. J. Owen, and J. L. Friedman, Phys. Rev. D 79, 124032 (2009), arXiv:0812.2163

[64] A. Bauswein and H.-T. Janka, Phys. Rev. Lett. 108, 011101 (2012), arXiv:1106.1616 [astro-ph.SR]

[65] A. Bauswein, H.-T. Janka, K. Hebeler, and A. Schwenk, Phys. Rev. D 86, 063001 (2012), arXiv:1204.1888 [astro-ph.SR]

[66] K. Takami, L. Rezzolla, and L. Baiotti, Phys. Rev. Lett. 113, 091104 (2014), arXiv:1403.5672 [gr-qc]

[67] J. M. Lattimer and F. D. Swesty, Nucl. Phys. A 535, 331 (1991)

[68] D. Radice, L. Rezzolla, and F. Galeazzi, Mon. Not. R. Astron. Soc. L. 437, L46 (2014), arXiv:1306.6052 [gr-qc]

[69] D. Radice, L. Rezzolla, and F. Galeazzi, Class. Quantum Grav. 31, 075012 (2014), arXiv:1312.5004 [gr-qc]

[70] D. Alic, C. Bona-Casas, C. Bona, L. Rezzolla, and C. Palenzuela, Phys. Rev. D 85, 064040 (2012), arXiv:1106.2254 [grqc]

[71] M. Shibata, K. Taniguchi, and K. Uryū, Phys. Rev. D 68 , 084020 (2003), gr-qc/0310030

[72] K. Takami, L. Rezzolla, and S. Yoshida, Mon. Not. R. Astron. Soc. 416, L1 (2011), arXiv:1105.3069 [gr-qc]

[73] P. Haensel, A. Y. Potekhin, and D. G. Yakovlev, Neutron Stars 1, 1st ed. (Springer-Verlag, New York, 2007)

[74] A. Drago and G. Pagliara, Physical Review C 92, 045801 (2015)

[75] I. Bombaci, D. Logoteta, I. Vidaña, and C. Providência, European Physical Journal A 52, 58 (2016), arXiv:1601.04559 [astro-ph.HE]
[76] G. Lugones, European Physical Journal A 52, 53 (2016), arXiv: 1508.05548 [astro-ph.HE]

[77] A. Drago and G. Pagliara, Phys. Rev. C 92, 045801 (2015), arXiv: 1506.08337 [nucl-th]

[78] N. K. Glendenning and C. Kettner, Astron. Astrophys. 353, L9 (2000), astro-ph/9807155

[79] I. N. Mishustin, M. Hanauske, A. Bhattacharyya, L. M. Satarov, H. Stöcker, and W. Greiner, Physics Letters B 552, 1 (2003), hep-ph/0210422

[80] J. Macher and J. Schaffner-Bielich, European Journal of Physics 26, 341 (2005), astro-ph/0411295

[81] M. G. Alford, G. F. Burgio, S. Han, G. Taranto, and D. Zappalà, Phys. Rev. D 92, 083002 (2015), arXiv:1501.07902 [nucl-th]

[82] A. Zacchi, M. Hanauske, and J. Schaffner-Bielich, Phys. Rev. D 93, 065011 (2016), arXiv:1510.00180 [nucl-th]

[83] C. Reisswig and D. Pollney, Class. Quantum Grav. 28, 195015 (2011), arXiv:1006.1632 [gr-qc]

[84] S. Wanajo, Y. Sekiguchi, N. Nishimura, K. Kiuchi, K. Kyutoku, and M. Shibata, Astrophys. J. 789, L39 (2014), arXiv:1402.7317 [astro-ph.SR]

[85] D. Radice, F. Galeazzi, J. Lippuner, L. F. Roberts, C. D. Ott, and L. Rezzolla, Mon. Not. R. Astron. Soc. 460, 3255 (2016), arXiv:1601.02426 [astro-ph.HE]

[86] A. Murguia-Berthier, G. Montes, E. Ramirez-Ruiz, F. De Colle, and W. H. Lee, Astrophys. J. 788, L8 (2014), arXiv: 1404.0383 [astro-ph.HE]

[87] A. Murguia-Berthier, E. Ramirez-Ruiz, G. Montes, F. De Colle, L. Rezzolla, S. Rosswog, K. Takami, A. Perego, and W. H. Lee, Astrophys. J. Lett. 835, L34 (2017), arXiv:1609.04828 [astro-ph.HE]

[88] G. Shen, C. J. Horowitz, and S. Teige, Physical Review C 82, 015806 (2010), arXiv:1004.0228 [nucl-th]

[89] G. Shen, C. J. Horowitz, and S. Teige, Physical Review C 83, 035802 (2011), arXiv:1101.3715 [astro-ph.SR]

[90] V. Mewes, F. Galeazzi, J. A. Font, P. J. Montero, and N. Stergioulas, Mon. Not. R. Astron. Soc. 461, 2480 (2016), arXiv:1605.02629 [astro-ph.HE]

[91] L. Bovard and L. Rezzolla, ArXiv e-prints (2017), arXiv: 1705.07882 [gr-qc]

[92] L. Baiotti, R. de Pietri, G. M. Manca, and L. Rezzolla, Phys. Rev. D 75, 044023 (2007), astro-ph/0609473

[93] G. M. Manca, L. Baiotti, R. DePietri, and L. Rezzolla, Class. Quantum Grav. 24, S171 (2007), arXiv:0705.1826

[94] K. D. Camarda, P. Anninos, P. C. Fragile, and J. A. Font, Astrophys. J. 707, 1610 (2009), arXiv:0911.0670 [astro-ph.SR]

[95] G. Corvino, L. Rezzolla, S. Bernuzzi, R. De Pietri, and B. Giacomazzo, Class. Quantum Grav. 27, 114104 (2010), arXiv:1001.5281 [gr-qc]

[96] B. Giacomazzo, L. Rezzolla, and N. Stergioulas, Phys. Rev. D 84, 024022 (2011), arXiv:1105.0122 [gr-qc]

[97] K. Kiuchi, K. Kyutoku, and M. Shibata, Phys. Rev. D 86 , 064008 (2012), arXiv:1207.6444 [astro-ph.HE]

[98] L. Franci, R. De Pietri, K. Dionysopoulou, and L. Rezzolla, Journal of Physics Conference Series 470, 012008 (2013), arXiv:1309.6549 [gr-qc]

[99] J. D. Kaplan, C. D. Ott, E. P. O'Connor, K. Kiuchi, L. Roberts, and M. Duez, Astrophys. J. 790, 19 (2014), arXiv:1306.4034 [astro-ph.HE]

[100] D. M. Siegel, R. Ciolfi, A. I. Harte, and L. Rezzolla, Phys. Rev. D R 87, 121302 (2013), arXiv:1302.4368 [gr-qc]

[101] D. M. Siegel, R. Ciolfi, and L. Rezzolla, Astrophys. J. 785, L6 (2014), arXiv:1401.4544 [astro-ph.HE]

[102] F. Löffler, R. De Pietri, A. Feo, F. Maione, and L. Franci, 
Phys. Rev. D 91, 064057 (2015), arXiv:1411.1963 [gr-qc]

[103] N. Stergioulas, A. Bauswein, K. Zagkouris, and H.-T. Janka, Mon. Not. R. Astron. Soc. 418, 427 (2011), arXiv: 1105.0368 [gr-qc]

[104] B. Margalit, B. D. Metzger, and A. M. Beloborodov, Phys. Rev. Lett. 115, 171101 (2015), arXiv:1505.01842 [astroph.HE]

[105] L.-X. Li and B. Paczynski, Astrophys. J. 507, L59 (1998), arXiv:astro-ph/9807272 [astro-ph]

[106] S. R. Kulkarni, astro-ph/0510256 (2005), astro-ph/0510256

[107] B. D. Metzger, G. Martínez-Pinedo, S. Darbha, E. Quataert, A. Arcones, D. Kasen, R. Thomas, P. Nugent, I. V. Panov, and N. T. Zinner, Mon. Not. R. Astron. Soc. 406, 2650 (2010), arXiv:1001.5029 [astro-ph.HE]

[108] L. F. Roberts, D. Kasen, W. H. Lee, and E. Ramirez-Ruiz, Astrophys. J. Lett. 736, L21 (2011), arXiv:1104.5504 [astroph.HE]

[109] D. Kasen, N. R. Badnell, and J. Barnes, Astrophys. J. 774, 25 (2013), arXiv:1303.5788 [astro-ph.HE]

[110] J. Barnes and D. Kasen, Astrophys. J. 775, 18 (2013), arXiv:1303.5787 [astro-ph.HE]

[111] M. Tanaka and K. Hotokezaka, Astrophys. J. 775, 113 (2013), arXiv:1306.3742 [astro-ph.HE]

[112] S. Rosswog, O. Korobkin, A. Arcones, F.-K. Thielemann, and T. Piran, Mon. Not. R. Astron. Soc. 439, 744 (2014), arXiv:1307.2939 [astro-ph.HE]

[113] D. Grossman, O. Korobkin, S. Rosswog, and T. Piran, Mon. Not. R. Astron. Soc. 439, 757 (2014), arXiv:1307.2943 [astroph.HE]

[114] J. Lippuner and L. F. Roberts, Astrophys. J. 815, 82 (2015), arXiv:1508.03133 [astro-ph.HE]

[115] V. Ravi and P. D. Lasky, Mon. Not. R. Astron. Soc. 441, 2433 (2014)

[116] E. P. Velikhov, Sov. Phys. JETP 9, 995 (1959)

[117] S. Chandrasekhar, Proc. Natl. Acad. Sci. 46, 253 (1960)

[118] S. A. Balbus and J. F. Hawley, Astrophys. J. 376, 214 (1991)

[119] O. Just, A. Bauswein, R. A. Pulpillo, S. Goriely, and H.-T. Janka, Mon. Not. R. Astron. Soc. 448, 541 (2015), arXiv:1406.2687 [astro-ph.SR]
[120] R. Ciolfi, W. Kastaun, B. Giacomazzo, A. Endrizzi, D. M. Siegel, and R. Perna, Phys. Rev. D 95, 063016 (2017), arXiv: 1701.08738 [astro-ph.HE]

[121] V. Paschalidis, Z. B. Etienne, and S. L. Shapiro, Phys. Rev. D 86, 064032 (2012), arXiv:1208.5487 [astro-ph.HE]

[122] K. Kiuchi, K. Kyutoku, Y. Sekiguchi, M. Shibata, and T. Wada, Phys. Rev. D 90, 041502 (2014), arXiv:1407.2660 [astro-ph.HE]

[123] K. Kiuchi, P. Cerdá-Durán, K. Kyutoku, Y. Sekiguchi, and M. Shibata, Phys. Rev. D 92, 124034 (2015), arXiv:1509.09205 [astro-ph.HE]

[124] K. Dionysopoulou, D. Alic, and L. Rezzolla, Phys. Rev. D 92 , 084064 (2015), arXiv:1502.02021 [gr-qc]

[125] C. Palenzuela, S. L. Liebling, D. Neilsen, L. Lehner, O. L. Caballero, E. O'Connor, and M. Anderson, Phys. Rev. D 92 , 044045 (2015), arXiv:1505.01607 [gr-qc]

[126] M. Ruiz, R. N. Lang, V. Paschalidis, and S. L. Shapiro, Astrophys. J. Lett. 824, L6 (2016), arXiv:1604.02455 [astro-ph.HE]

[127] T. Kawamura, B. Giacomazzo, W. Kastaun, R. Ciolfi, A. Endrizzi, L. Baiotti, and R. Perna, Phys. Rev. D 94, 064012 (2016), arXiv:1607.01791 [astro-ph.HE]

[128] D. J. Price and S. Rosswog, Science 312, 719 (2006), astro$\mathrm{ph} / 0603845$

[129] B. Giacomazzo, L. Rezzolla, and L. Baiotti, Phys. Rev. D 83, 044014 (2011)

[130] B. Giacomazzo, L. Rezzolla, and L. Baiotti, Mon. Not. R. Astron. Soc. 399, L164 (2009), arXiv:0901.2722 [gr-qc]

[131] W. E. East, V. Paschalidis, F. Pretorius, and S. L. Shapiro, Phys. Rev. D 93, 024011 (2016), arXiv:1511.01093 [astroph.HE]

[132] D. Radice, S. Bernuzzi, and C. D. Ott, Phys. Rev. D 94 064011 (2016), arXiv:1603.05726 [gr-qc]

[133] L. Lehner, S. L. Liebling, C. Palenzuela, and P. M. Motl, Phys. Rev. D 94, 043003 (2016), arXiv:1605.02369 [gr-qc]

[134] F. Maione, R. De Pietri, A. Feo, and F. Löffler, Classical and Quantum Gravity 33, 175009 (2016), arXiv:1605.03424 [grqc]

[135] A. Feo, R. De Pietri, F. Maione, and F. Löffler, Class. Quantum Grav. 34, 034001 (2017), arXiv:1608.02810 [gr-qc] 